\title{
Moduli of continuity, isoperimetric profiles, and multi-point estimates in geometric heat equations
}

\author{
Ben Andrews
}

Dedicated to Richard, Leon and Karen, for showing us how it should be done.

\begin{abstract}
Estimates on moduli of continuity, isoperimetric profiles of various kinds, and quantities involving function values at several points have been central in several recent results in geometric analysis. This paper surveys some of the techniques and their applications.
\end{abstract}

\section{Introductory comments}

In this article I want to describe some techniques which have been applied with some success recently to a variety of problems, ranging from my proof with Julie Clutterbuck of the sharp lower bound on the fundamental gap for Schrödinger operators [8] to Brendle's proof of the Lawson conjecture [29] and my proof with Haizhong Li of the Pinkall-Sterling conjecture [17]. I will discuss several other interesting applications below. The common thread in these techniques is the application of the maximum principle to functions involving several points or to functions depending on the global structure of solutions. Such techniques are not entirely new: In the analysis of parabolic equations in one variable, functions involving two points have been used extensively, going back to the work of Kružkov [61]. Later work using related ideas include the 'concavity maximum principle' of Korevaar [59], who considered the function

$$
\mathcal{C}(x, y, \mu)=u(\mu y+(1-\mu) x)-\mu u(y)-(1-\mu) u(x)
$$

for $\mu \in(0,1)$ and $x, y$ in the domain of $u$, with $u$ satisfying some elliptic or parabolic equation. He used a maximum principle to prove non-positivity of $\mathcal{C}$, implying convexity of the function $u$, for several situations of interest. Korevaar's argument was later extended by several authors, notably Kawohl

This survey describes work supported by Discovery Projects grants DP0985802, DP120102462 and DP120100097 of the Australian Research Council.

(C) 2015 International Press 
[55] and Kennington [56,57]. I will mention later some contributions of Hamilton [51,52] and Huisken [54] where related ideas were developed for applications in geometric flow problems. It should be pointed out also that very similar ideas arise in the theory of viscosity solutions as expounded by Crandall, Ishii and Lions [41] — for example the proof of the comparison principle there is based on the use of a two-point function to characterise semi-jets of a non-smooth function, and the basic computations involved are similar to some of those I will employ below.

\section{Preserving modulus of continuity in heat equations}

Perhaps the simplest example of applying the maximum principle to a function of several points arises in controlling the modulus of continuity of a solution of a parabolic equation. Consider a smooth solution of the heat equation in $\mathbb{R}^{n}$, either on a domain with suitable boundary conditions (Neumann is simplest but others can also be handled) or otherwise with good control - for simplicity let us consider here only the case where the solution is spatially periodic in some lattice. We will consider mostly the heat equation, but the techniques work well also for a wider class of parabolic equations, which might be described as 'quasilinear equations with gradientdependent coefficients'. In particular these are quasilinear flows which are invariant under rigid motions of space, and vertical translation of the graph.

In the Euclidean setting these equations preserve any initial modulus of continuity: More generally, for any fixed vector $w$, any inequality of the form $u(x+w)-u(x) \leq f(w)$ is preserved (that is, if the inequality holds for all $x$ at an initial time, it continues to hold for all $x$ for all subsequent times). To see this, we simply observe that the function $u(x+w)-f(w)$ is a solution of the same equation as $u$ (since it is obtained by translation and adding a constant), so this remains less than $u$ if it is initially so. A modulus of continuity is simply given by estimates of this form for every $w$, with $f$ depending only on the length of $w$.

Now we consider the problem of improving a modulus of continuity: For simplicity let us consider a somewhat restricted class of flows, the 'isotropic' flows of the form

$$
\frac{\partial u}{\partial t}=\mathcal{L}[u]:=\left[a(|D u|) \frac{u_{i} u_{j}}{|D u|^{2}}+b(|D u|)\left(\delta^{i j}-\frac{u_{i} u_{j}}{|D u|^{2}}\right)\right] D_{i} D_{j} u,
$$

so that $a$ determines the rate of diffusion in the gradient directions, and $b$ the diffusion in the orthogonal directions. These examples include the classical heat equation (with $a=b=1$ ), the $p$-Laplacian heat equations (with $a=(p-1)|D u|^{p-2}$ and $b=|D u|^{p-2}$ and the graphical mean curvature flow (with $a=\frac{1}{1+|D u|^{2}}$ and $b=1$ ).

Given a function $u$, the modulus of continuity $\omega$ of $u$ can be defined by

$$
\omega(s)=\sup \left\{\frac{u(y)-u(x)}{2} \mid \frac{|y-x|}{2}=s\right\}
$$


(the factors of 2 are different from the usual definition, and are included for later convenience, in particular to arrange the nice statement of Proposition 1). As $u$ evolves, what can we say about the modulus of continuity at later times? There is a pretty answer, encapsulated by the following:

Proposition 1. If $u: \mathbb{R}^{n} \times[0, T) \rightarrow \mathbb{R}$ is a (spatially periodic) smooth solution of (1), then the modulus of continuity

$$
\omega(s, t)=\sup \left\{\frac{u(y, t)-u(x, t)}{2} \mid \frac{|y-x|}{2}=s\right\}
$$

is a viscosity subsolution of the one-dimensional heat equation $\omega_{t}=a\left(\omega^{\prime}\right) \omega^{\prime \prime}$ on $(0, \infty) \times(0, T)$.

Proof. Don't be put off by the 'viscosity subsolution' terminology: This is a very simple way to define a subsolution in a situation where the function may be non-smooth (which could be the case for the modulus of continuity since it is defined as a supremum), and amounts to the following: We must show that if $\varphi$ is a smooth function that touches $\omega$ from above, in the sense that $\varphi(s, t) \geq \omega(s, t)$ for all $s$ near $s_{0}$ and $t \leq t_{0}$ near $t_{0}$, and equality holds at $\left(s_{0}, t_{0}\right)$, then $\varphi$ must satisfy

$$
\varphi_{t} \leq a\left(\varphi^{\prime}\right) \varphi^{\prime \prime}
$$

at the point $\left(s_{0}, t_{0}\right)$. To prove this for some $s_{0}>0$ and $t_{0}>0$, we note that by the definition of $\omega$ the following inequality holds:

$$
\varphi\left(\frac{|y-x|}{2}, t\right) \geq \omega\left(\frac{|y-x|}{2}, t\right) \geq \frac{u(y, t)-u(x, t)}{2}
$$

for all points $x, y \in \mathbb{R}^{n}$ with $|y-x|$ close to $s_{0}$ and $t \leq t_{0}$ close to $t_{0}$. Furthermore, since $\varphi\left(s_{0}, t_{0}\right)=\omega\left(s_{0}, t_{0}\right)$, and since we are in a co-compact situation, there exist points $x_{0}$ and $y_{0}$ with $\left|y_{0}-x_{0}\right|=2 s_{0}$ attaining the supremum, so that $\varphi\left(\frac{\left|x_{0}-y_{0}\right|}{2}, t_{0}\right)=\omega\left(s_{0}, t_{0}\right)=\frac{1}{2}\left(u\left(y_{0}, t_{0}\right)-u\left(x_{0}, t_{0}\right)\right)$. Now the functions on the left and the right are both smooth (even though the function $\omega$ in the middle may not be), so we can apply the first and second derivative tests to deduce the following: Differentiating in the time direction gives

$$
\left.\frac{\partial \varphi}{\partial t}\right|_{\left(s_{0}, t_{0}\right)} \leq \frac{1}{2}\left(\left.\frac{\partial u}{\partial t}\right|_{\left(y_{0}, t_{0}\right)}-\left.\frac{\partial u}{\partial t}\right|_{\left(x_{0}, t_{0}\right)}\right)
$$

while differentiating with respect to $x$ gives

$$
-\varphi^{\prime}\left(s_{0}, t_{0}\right) \frac{y_{0}-x_{0}}{\left|y_{0}-x_{0}\right|}=-\nabla u\left(x_{0}, t_{0}\right)
$$

and differentiating in $y$ gives

$$
\varphi^{\prime}\left(s_{0}, t_{0}\right) \frac{y_{0}-x_{0}}{\left|y_{0}-x_{0}\right|}=\nabla u\left(y_{0}, t_{0}\right) .
$$

In particular the spatial gradients $\nabla u\left(x_{0}, t_{0}\right)$ and $\nabla u\left(y_{0}, t_{0}\right)$ are equal, with magnitude $\varphi^{\prime}$ and pointing in the direction from $x_{0}$ to $y_{0}$. To make use of 
this observation we choose our basis for $\mathbb{R}^{n}$ so that $e_{n}=\frac{y_{0}-x_{0}}{\left|y_{0}-x_{0}\right|}$. Then we observe that at $\left(x_{0}, t_{0}\right)$ and $\left(y_{0}, t_{0}\right)$ we have

$$
\frac{\partial u}{\partial t}=a\left(\varphi^{\prime}\right) u_{n n}+b\left(\varphi^{\prime}\right) \sum_{i<n} u_{i i}
$$

where the subscripts denote partial derivatives. In particular the timederivative inequality (3) becomes

(6)

$$
2 \frac{\partial \varphi}{\partial t} \leq a\left(\varphi^{\prime}\right)\left(u_{n n}\left(y_{0}, t_{0}\right)-u_{n n}\left(x_{0}, t_{0}\right)\right)+b\left(\varphi^{\prime}\right) \sum_{i<n}\left(u_{i i}\left(y_{0}, t_{0}\right)-u_{i i}\left(x_{0}, t_{0}\right)\right) .
$$

We also obtain a second derivative inequality from taking the second derivative along any curve $(x(s), y(s))$ passing through $\left(x_{0}, y_{0}\right)$. To obtain useful inequalities we choose particular variations which produce the terms arising in (6): For each $i<n$ we consider $(x(s), y(s))=\left(x_{0}+s e_{i}, y_{0}+s e_{i}\right)$, along which $|y(s)-x(s)|$ does not change, so we find

$$
0 \geq u_{i i}\left(y_{0}, t_{0}\right)-u_{i i}\left(x_{0}, t_{0}\right) \text {. }
$$

In the $e_{n}$ direction we need to choose a variation which will produce the term $\varphi^{\prime \prime}$, so we choose $(x(s), y(s))=\left(x_{0}-s e_{n}, y_{0}+s e_{n}\right)$. Then we have $\frac{\partial}{\partial s}|y(s)-x(s)|=2$ and $\frac{\partial^{2}}{\partial s^{2}}|y(s)-x(s)|=0$, so that

$$
2 \varphi^{\prime \prime}\left(s_{0}, t_{0}\right) \geq u_{n n}\left(y_{0}, t_{0}\right)-u_{n n}\left(x_{0}, t_{0}\right) .
$$

Finally, combining the inequalities (6), (7) and (8) gives

$$
\frac{\partial \varphi}{\partial t} \leq a\left(\varphi^{\prime}\right) \varphi^{\prime \prime}
$$

as required, and we have proved that $\omega_{t} \leq a\left(\omega^{\prime}\right) \omega^{\prime \prime}$ in the viscosity sense.

COROllary 2. If $u$ is a spatially periodic solution of (1), and $\varphi$ satisfies $\varphi_{t} \geq a\left(\varphi^{\prime}\right) \varphi^{\prime \prime}$ on $(0, \infty) \times(0, T)$ with $\varphi(0, t) \geq 0$ and $\varphi^{\prime} \geq 0$, and $|u(y, 0)-u(x, 0)| \leq 2 \varphi\left(\frac{|y-x|}{2}, 0\right)$ for all $x \neq y$, then $|u(y, t)-u(x, t)| \leq$ $2 \varphi\left(\frac{|y-x|}{2}, t\right)$ for all $x, y$ and $t \geq 0$.

That is, if the initial data $\varphi(., 0)$ lies above the modulus of continuity of $u(., 0)$, and we evolve $\varphi$ by the one-dimensional heat equation $\varphi_{t}=a\left(\varphi^{\prime}\right) \varphi^{\prime \prime}$, then $\varphi(., t)$ lies above the modulus of continuity of $u(., t)$ for each positive $t$. The proof is immediate from Proposition 1 , since the function $\varphi_{\varepsilon}=\varphi+\varepsilon \mathrm{e}^{t}$ satisfies $\partial_{t} \varphi_{\varepsilon}>a\left(\varphi_{\varepsilon}^{\prime}\right) \varphi_{\varepsilon}^{\prime \prime}$, and so cannot touch $\omega$ from above.

This result has a nice interpretation which also demonstrates that the result is sharp: A particularly simple class of solutions of (1) (which I will call 'one-dimensional solutions') are those where the solution only depends on one variable in $\mathbb{R}^{n}$, in which case it has the form $u\left(x_{1}, \ldots, x^{n}\right)=\varphi\left(x^{n}\right)$, where $\varphi_{t}=a\left(\varphi^{\prime}\right) \varphi^{\prime \prime}$. In particular, if $\varphi(., 0)$ is odd and is concave and positive for positive $x$, then this is true also for positive times, and one can check that the modulus of continuity $\omega$ of this solution is exactly $\omega(s, t)=\varphi(s, t)$, 
with equality occurring at all points of the form $x_{0}=\left(x^{1}, x^{2}, \ldots, x^{n-1},-x^{n}\right)$ and $y_{0}=\left(x^{1}, \ldots, x^{n-1}, x^{n}\right)$ and any $t \geq 0$. Thus the corollary says precisely that if the modulus of continuity of the initial data $u_{0}$ is bounded by the modulus of continuity of the one-dimensional solution, then this remains true for all positive times - the result becomes a comparison theorem between the modulus of continuity of an arbitrary solution and that of a special symmetric solution. This is a phenomenon we will see repeated over and over in different settings ${ }^{1}$. As a particular application, for a solution of the heat equation which is initially bounded (so that $u(y, 0)-u(x, 0) \leq M$ for all $x$ and $y$ for some constant $M)$ we can choose $\varphi(s, 0)=\frac{M}{2}$ and solve the one-dimensional heat equation to get

$$
|u(y, t)-u(x, t)| \leq M \operatorname{erf}\left(\frac{|y-x|}{4 \sqrt{t}}\right)
$$

for all $x, y$. In particular this gives the Lipschitz bound $|\nabla u(x, t)| \leq \frac{M}{2 \sqrt{\pi t}}$ for all $x$ and all $t>0$. This estimate is sharp, with equality holding for the error function one-dimensional solution. Similarly, for bounded solutions of the $p$-Laplacian heat flow the optimal modulus of continuity is that of the one-dimensional solution $u(x, t)=\left(\frac{p-2}{2 p(p-1)}\right)^{\frac{1}{p-2}} \int_{0}^{x / t^{1 / p}}\left(1-\xi^{2}\right)_{+}^{\frac{1}{p-2}} d \xi$ for $p>2$, or $u(x, t)=\left(\frac{2 p(p-1)}{2-p}\right)^{\frac{1}{2-p}} \int_{0}^{x / t^{1 / p}} \frac{1}{\left(1+\xi^{2}\right)^{1 /(2-p)}} d \xi$ for $1<p<2$, and this also determines the sharp gradient estimate in this situation:

$$
|\nabla u(x, t)| \leq C_{p} \frac{M^{2 / p}}{t^{1 / p}} .
$$

\section{Heat equations on manifolds}

Moving from the context of Euclidean domains to domains in a Riemannian manifold $(M, g)$ is mostly a straightforward matter: One simply uses the Riemannian distance function $d(x, y)=\inf \{L[\gamma] \mid \gamma:[0,1] \rightarrow$ $M, \gamma(0)=x, \gamma(1)=y\}$ to define the modulus of continuity. Of course $d$ is not a smooth function in general, but it does satisfy useful second derivative inequalities (in the viscosity sense) provided the Ricci curvature is controlled from below, as I will explain ${ }^{2}$.

The modulus of continuity $\omega$ of a smooth function $u$ on $M$ is defined as before by

$$
\omega(s):=\sup \left\{\frac{u(y)-u(x)}{2} \mid \frac{d(x, y)}{2}=s\right\}
$$

\footnotetext{
${ }^{1}$ The essential computations involved in the results described above appeared first in Julie Clutterbuck's $\mathrm{PhD}$ thesis [39], and in our joint papers [6,7]. The results hold also for boundary value problems on convex domains, and also for a wider class of equations than the isotropic ones considered here.

${ }^{2}$ The estimate of modulus of continuity for heat equations on manifolds first appeared in [9] and [68].
} 
In order to formulate the correct equation to be satisfied (in the viscosity sense) by the modulus of continuity, it is useful to identify the 'onedimensional' solutions which are relevant. These are given by a 'warped product' description of the constant sectional curvature $K$ model spaces $\bar{M}_{K}$, given by starting with a totally geodesic hypersurface $\Sigma$ and taking the embedding $X: \Sigma \times I \rightarrow \bar{M}_{K}$ given by $X(z, s)=\exp (s \nu(z))$, where $\nu$ is a unit normal vector to $\Sigma$. This induces the Riemannian metric $d s^{2}+C_{K}(s)^{2} \bar{g}$, where $\bar{g}$ is the Riemannian metric on $\Sigma$, and $C_{K}(s)$ is defined by $C_{K}^{\prime \prime}+K C_{K}=0, C_{K}(0)=1$ and $C_{K}^{\prime}(0)=0$. The 'one-dimensional' solutions of (1) are those which depend only on $s$ and not on $z$. The equation then reduces to a one-dimensional heat equation in $s$ and $t$, as follows: Since $u$ is a function of $s$ only, we have $u_{i j}=u^{\prime} s_{i j}+u^{\prime \prime} s_{i} s_{j}$. If we choose coordinates such that $e_{n}=\partial_{s}$ and $e_{i}$ is tangent to the level set $\Sigma_{s}=X(\Sigma, s)$ for $i<n$, then this gives $u_{n n}=u^{\prime \prime}, u_{i n}=0$, and $u_{i j}=u^{\prime} s_{i j}$ (here subscripts are covariant derivatives). Along $\Sigma_{s}$, the second covariant derivatives of $s$ coincide with the second fundamental form of $\Sigma_{s}$. This is equal to half of the derivative in $s$ of the metric, so equals $T_{K}$ times the metric, where $T_{K}=\frac{C_{K}^{\prime}}{C_{K}}$. This gives $u_{t}=\left[a(|D u|) \frac{u_{i} u_{j}}{|D u|^{2}}+b(|D u|)\left(\delta_{i j}-\frac{u_{i} u_{j}}{|D u|^{2}}\right)\right] u_{i j}=$ $a\left(u^{\prime}\right) u^{\prime \prime}+(n-1) T_{K}(s) b\left(u^{\prime}\right) u^{\prime}$. We also note that if $u(., t)$ is odd and concave and increasing for positive $s$, then $\omega(., t)=u(., t)$, so in such examples the modulus of continuity itself satisfies the equation we just derived. For arbitrary (not necessarily symmetric) solutions we then have the following result:

Proposition 3. If $u: M \times[0, T) \rightarrow \mathbb{R}$ is a solution of (1) on a compact manifold $M$ with diameter $D$ (with convex or empty boundary with Neumann boundary conditions), then the modulus of continuity $\omega:[0, D / 2] \times[0, T) \rightarrow$ $\mathbb{R}$ satisfies

$$
\omega_{t} \leq a\left(\omega^{\prime}\right) \omega^{\prime \prime}+(n-1) T_{K} b\left(\omega^{\prime}\right) \omega^{\prime}
$$

in the viscosity sense.

Proof. If $\varphi$ is a smooth function which lies above $\omega$ for $(s, t)$ near $\left(s_{0}, t_{0}\right)$, with equality at $\left(s_{0}, t_{0}\right)$, then we have for any smooth path $\gamma$ : $[0,1] \rightarrow M$ the inequality

$$
u(\gamma(1), t)-u(\gamma(0), t)-2 \varphi\left(\frac{L[\gamma]}{2}, t\right) \leq 0
$$

for each $t \leq t_{0}$ close to $t_{0}$, provided $L[\gamma]$ is close to $s_{0}$. Since equality holds at $\left(s_{0}, t_{0}\right)$, we have points $y_{0}$ and $x_{0}$ in $M$ with $d\left(x_{0}, y_{0}\right)=2 s_{0}$, such that $u\left(y_{0}, t_{0}\right)-u\left(x_{0}, t_{0}\right)=2 \varphi\left(\frac{d\left(x_{0}, t_{0}\right)}{2}, t_{0}\right)$, and if $\gamma_{0}$ is a length-minimising geodesic from $x_{0}$ to $y_{0}$ then we have $u\left(y_{0}, t_{0}\right)-u\left(x_{0}, t_{0}\right)=2 \varphi\left(\frac{L\left[\gamma_{0}\right]}{2}, t_{0}\right)$ (that is, equality holds in (10) at $\left.\gamma_{0}\right)$. We can now deduce inequalities from the first and second derivative tests along any smooth family of curves $\gamma_{r}$ passing through $\gamma_{0}$. This requires a computation of the first and second variation of 
length under such variations, which is rather standard: If $\gamma:(r, s) \mapsto \gamma_{r}(s)$ is smooth, then we have

$$
\begin{aligned}
\left.\frac{\partial}{\partial r} L\left[\gamma_{r}\right]\right|_{r=0} & =\int_{0}^{1} \frac{\partial}{\partial r}\left|\gamma_{s}\right| d s \\
& =\int_{0}^{1} g\left(T, \nabla_{r} \gamma_{s}\right) d s=\int_{0}^{1} \frac{\partial}{\partial s} g\left(T, \gamma_{r}\right) d s=\left.g\left(T, \gamma_{r}\right)\right|_{0} ^{1},
\end{aligned}
$$

where $T$ is the unit tangent vector to $\gamma_{0}$. The second derivative is given by

$$
\left.\frac{\partial^{2}}{\partial r^{2}} L\left[\gamma_{r}\right]\right|_{r=0}=\frac{1}{L} \int_{0}^{1}\left|\left(\nabla_{s} \gamma_{r}\right)^{\perp}\right|^{2}-R\left(\gamma_{s}, \gamma_{r}, \gamma_{s}, \gamma_{r}\right) d s+\left.g\left(T, \nabla_{r} \gamma_{r}\right)\right|_{0} ^{1}
$$

The first derivatives at $r=0$ for an arbitrary smooth variation of curves in (10) give the following:

$$
\left\langle\nabla u\left(y_{0}, t_{0}\right)-\varphi^{\prime} \gamma_{0}^{\prime}(1), \gamma_{r}(1)\right\rangle-\left\langle\nabla u\left(x_{0}, t_{0}\right)-\varphi^{\prime} \gamma_{0}^{\prime}(0), \gamma_{r}(0)\right\rangle=0,
$$

from which we conclude that $\nabla u\left(y_{0}, t_{0}\right)=\varphi^{\prime} \gamma_{0}^{\prime}(1)$ and $\nabla u\left(x_{0}, t_{0}\right)=\varphi^{\prime} \gamma_{0}^{\prime}(0)$. Now we consider the inequalities obtained from second variations. A guiding principle is that in order to obtain a sharp result, any inequalities that we apply must hold with equality in the model 'one-dimensional' case. In that case, equality holds in (10) when $\gamma$ has the form $\gamma(s)=X\left(z, \frac{L(2 s-1)}{2}\right)$ for fixed $z$ and $L$, so in order to produce inequalities which are sharp (more precisely, which hold in the model case) we should consider variations $\gamma(r, s)$ which correspond to moving through families of curves of this form. In order to select the correct variations it is useful to work in 'Fermi coordinates' along the geodesic $\gamma_{0}$ : Choose an orthonormal basis $\left\{e_{i}\right\}$ to $T_{x_{0}} M$ (with respect to $\left.g_{t_{0}}\right)$ with $e_{n}$ equal to the unit tangent vector $T(0)$ of $\gamma_{0}$ at $x_{0}$. Then parallel transport along $\gamma_{0}$ to produce an orthonormal basis $\left\{e_{i}(s)\right\}$ for each tangent space $T_{\gamma_{0}(s)} M$. Observe that $e_{n}(s)=T(s)$ for each $s$.

One natural variation in the model case is to keep $z$ fixed and increase $L$, so that $\gamma(r, s)=\gamma_{0}\left(s+r \frac{2 s-1}{L}\right)$ and $\gamma_{r}=\frac{2 s-1}{L} \gamma_{0}^{\prime}$. Then (12) gives $\partial_{r} L=2$ and $\partial_{r}^{2} L=0$. Note that $\gamma_{r}(0)=-T(0)=-e_{n}(0)$ and $\gamma_{r}(1)=T(1)=e_{n}(1)$, so that the second derivative inequality becomes

$$
u_{n n}\left(y_{0}, t_{0}\right)-u_{n n}\left(x_{0}, t_{0}\right)-2 \varphi^{\prime \prime} \leq 0,
$$

exactly as in (8), where the subscripts now denote covariant derivatives in directions corresponding to the basis $\left\{e_{i}\right\}$. Other cases correspond to varying $z$ and keeping $L$ fixed. In this case the variation vector field $\gamma_{r}$ is a Jacobi field of the model space, which we can take to be given by $\gamma_{r}(s)=\frac{C_{K}(L(2 s-1) / 2)}{C_{K}(L / 2)} e_{i}$, for some $i<n$. Then $\partial_{r} L=0$, and (12) gives

$$
\partial_{r}^{2} L=\frac{2 C_{K}^{\prime}(L / 2)}{C_{K}(L / 2)}-\int_{-L / 2}^{L / 2} \frac{C_{K}(x)^{2}}{C_{K}(L / 2)^{2}}\left[R\left(e_{i}, T, e_{i}, T\right)-K\right] d x,
$$


after changing variable to $x=\frac{L(2 s-1)}{2}$, and an integration by parts. This produces the following second derivative inequality:

$$
\begin{aligned}
u_{i i}\left(y_{0}, t_{0}\right) & -u_{i i}\left(x_{0}, t_{0}\right) \\
& -\varphi^{\prime}\left(2 T_{K}(L / 2)-\int_{-L / 2}^{L / 2} \frac{C_{K}(x)^{2}}{C_{K}(L / 2)^{2}}\left[R\left(e_{i}, T, e_{i}, T\right)-K\right] d x\right) \leq 0 .
\end{aligned}
$$

Adding this inequality over $i$ between 1 and $n-1$ we obtain

$$
\begin{aligned}
& \sum_{i<n}\left(\left.u_{i i}\right|_{\left(y_{0}, t_{0}\right)}-\left.u_{i i}\right|_{\left(x_{0}, t_{0}\right)}\right)-2(n-1) T_{K}\left(\frac{L}{2}\right) \varphi^{\prime} \\
& \quad+\varphi^{\prime} \int_{-\frac{L}{2}}^{\frac{L}{2}} \frac{C_{K}(x)^{2}}{C_{K}\left(\frac{L}{2}\right)^{2}}[\operatorname{Rc}(T, T)-(n-1) K] d x \leq 0 .
\end{aligned}
$$

The assumption that $\mathrm{Rc} \geq(n-1) K g$ guarantees that the last term is nonnegative and can be discarded. Adding this inequality multiplied by $b\left(\varphi^{\prime}\right)$ with (13) multiplied by $a\left(\varphi^{\prime}\right)$ yields

$$
\left.\mathcal{L}[u]\right|_{\left(y_{0}, t_{0}\right)}-\left.\mathcal{L}[u]\right|_{\left(x_{0}, t_{0}\right)}-2 a\left(\varphi^{\prime}\right) \varphi^{\prime \prime}-2(n-1) T_{K} b\left(\varphi^{\prime}\right) \varphi^{\prime} \leq 0 .
$$

On the other hand the time derivative inequality yields

$$
\left.\mathcal{L}[u]\right|_{\left(y_{0}, t_{0}\right)}-\left.\mathcal{L}[u]\right|_{\left(x_{0}, t_{0}\right)}-2 \varphi_{t} \geq 0 .
$$

These two inequalities combine to give

$$
\varphi_{t} \leq a\left(\varphi^{\prime}\right) \varphi^{\prime \prime}+(n-1) T_{K} b\left(\varphi^{\prime}\right) \varphi^{\prime}
$$

which proves that (9) holds in the viscosity sense.

The sharpness of the estimate may be observed as follows: Given a solution of $(1)$, with modulus of continuity $\omega\left(., t_{0}\right)$ at some time $t_{0}$, construct a symmetric solution $\bar{u}$ for $t \geq t_{0}$ with initial data at time $t_{0}$ given by $\bar{u}\left(z, s, t_{0}\right)=\omega\left(s, t_{0}\right)$. This evolves to remain symmetric, so of the form $\bar{u}(z, s, t)=\varphi(s, t)$ where $\varphi$ satisfies (18), and $\bar{u}(., t)$ has modulus of continuity equal to $\varphi(., t)$. Thus Proposition 3 may be interpreted as saying that the modulus of continuity of an arbitrary solution of (1) evolves to be no greater than that of the symmetric solution with the same initial modulus of continuity.

The technique given here also applies in various other situations: The paper [19] with Lei $\mathrm{Ni}$ treats the situation of the drift-Laplacian heat flow in Bakry-Émery manifolds with a lower bound on the Bakry-Émery Ricci tensor, and [21] (written with Sijian Tang, who was at the time an undergraduate student at Tsinghua University) treats the more general situation of heat flows corresponding to operators satisfying a $C D(n, K)$ condition. 


\section{Eigenvalue lower bounds}

The initial motivation of the modulus of continuity estimates as developed in $[6,7,39]$ was to provide bounds for positive times on the gradient of solutions, depending only on an initial oscillation bound. This works surprisingly well: For example, one can prove gradient estimates for graphical solutions of smoothly anisotropic mean curvature flows by this method $[7$, Theorems 6,10$]$, whereas this has not yet been accomplished using direct estimates on the gradient except in special cases [40]. However, it has turned out that some of the most important applications arise from the large time implications of the modulus of continuity estimates. These were developed in $[8,9,68]$. The basic idea is very simple, at least in the case of the heat equation (on a compact manifold, or a domain with convex boundary and Neumann boundary condition): Decomposing the initial data as a sum of eigenfunctions of the Laplacian, we find that the solution has the form

$$
u(x, t)=\sum_{i \geq 0} a_{i} \mathrm{e}^{-\lambda_{i} t} \varphi_{i}(x),
$$

where $\varphi_{i}$ is the $i$ th eigenfunction of the Laplacian, satisfying $\Delta \varphi_{i}+\lambda_{i} \varphi_{i}=0$, and we assume $\lambda_{i}$ is non-decreasing in $i$. The term with $i=0$ corresponds to constant functions with $\lambda_{0}=0$, and the first non-trivial Neumann eigenvalue is $\lambda_{1}>0$. As $t$ becomes large, this means that $u$ is well approximated by $a_{0}+a_{1} \mathrm{e}^{-\lambda_{1} t} \varphi_{1}(x)$, and in particular the oscillation of $u(., t)$ is bounded by $C \mathrm{e}^{-\lambda_{1} t}$ for some $C$. Conversely, if we can prove that arbitrary smooth solutions have oscillation decaying at a rate $C \mathrm{e}^{-\sigma t}$, then we can deduce that $\lambda_{1} \geq \sigma$ : The particular solution given by the first eigenfunction decays at rate $\mathrm{e}^{-\lambda_{1} t}$, and this is not bounded by $C \mathrm{e}^{-\sigma t}$ for large $t$ if $\lambda_{1}<\sigma$.

The key observation is that the modulus of continuity estimate leads directly to an exponential decay rate for the oscillation of solutions of the heat equation, implying a lower bound on the first eigenvalue: Proposition 3 implies that the modulus of continuity, and hence the oscillation, of an arbitrary solution $u$ of (1) is bounded by that of a solution of the one-dimensional model equation (18), which decays at an exponential rate $C \mathrm{e}^{-\bar{\lambda}_{1} t}$, where $\bar{\lambda}_{1}$ is the first eigenvalue of the operator $L:=\frac{\partial^{2}}{\partial s^{2}}+2(n-$ 1) $T_{K} \frac{\partial}{\partial s}$ on the interval $[-D / 2, D / 2]$ with Neumann conditions. It follows that $\lambda_{1} \geq \bar{\lambda}_{1}$. Note that $\bar{\lambda}_{1}$ depends only on the lower bound on Ricci curvature $K$, the diameter $D$ of the domain, and the dimension $n$. The estimate is sharp but not attained: The eigenvalue $\bar{\lambda}_{1}$ holds exactly for a domain which is symmetric, such as $\left\{X(z, s):|s|<D / 2, z \in B_{r}\left(z_{0}\right)\right\}$. This domain satisfies the required lower Ricci curvature bound, but has diameter larger than $D$ but approaching $D$ as $r \rightarrow 0$. That is, the lower bound $\lambda_{1} \geq \bar{\lambda}_{1}(D, K, n)$ is the best possible estimate in terms of $D, K$ and $n$.

I emphasise that this optimal lower eigenvalue bound is not new: The result in the case of non-negative Ricci curvature $(K=0)$ was proved by 
Zhong and Yang [80]. The result for general $K$ has been proved previously by $\mathrm{Mu}-\mathrm{Fa}$ Chen and Feng-Yu Wang $[37,38]$ who applied the probabilistic 'coupling method' to the Brownian motion associated with heat flow, and independently by Pawel Kröger [60] using gradient estimates. All of these methods have their roots in the arguments of $\mathrm{Li}$ and Yau $[63,64]$. The results (also using gradient estimates) were also encapsulated in the results in a more general setting of Bakry and Qian [25]. The advantage of the approach outlined above using the modulus of continuity is that it is very direct and simple and uses nothing more than the maximum principle.

See $[19,21]$ for the derivation of optimal lower eigenvalue bounds for operators satisfying $C D(n, K)$ conditions, including the Bakry-Émery case, using modulus of continuity estimates.

\section{Harmonic map heat flows}

Another situation where the modulus of continuity estimates apply to give simpler and cleaner proofs of known results is the harmonic map heat flow into targets with non-positive sectional curvatures. The well-known result of Eells and Sampson [42] is that any (smooth, or more generally finite energy) map $u_{0}$ from a compact Riemannian manifold $(M, g)$ to a manifold of non-positive sectional curvature $(N, h)$ deforms under harmonic map heat flow $u_{t}=\Delta u$ through a family of maps $u(., t)$ to a harmonic map $u_{\infty}$ as time approaches infinity. Here $\Delta u$ is the map Laplacian, produced from the Levi-Civita connection of $g$ on $T M$ and the pull-back connection on the pull-back bundle $u^{*} T N$ coming from the Levi-Civita connection of $h$ on $T N$. In the case that $N$ is isometrically embedded as a submanifold of a Euclidean space, this is given by computing the Laplacian of $u$ as a map into the Euclidean background using the Levi-Civita connection on $M$, and then taking the orthogonal projection onto the tangent space of $N$.

The modulus of continuity gives a simpler argument for one of the technically most involved parts of the proof of the Eells-Sampson theorem: The proof of a bound on the energy density $e(u)=|D u|^{2}$. Once this bound is obtained, bounds on all higher derivatives of $u$ follow by standard bootstrapping arguments. But obtaining a bound on $e(u)$ (assuming only an initial energy bound) requires some analytic work, such as application of a Moser iteration scheme or some equivalent argument to move from the $L^{2}$ energy bound to the pointwise bound on energy density.

In recent work with my PhD student David Shellard [20], we proved this more directly using the modulus of continuity argument, as follows: The modulus of continuity $\omega$ of a map $u: M \rightarrow N$ is defined by

$$
\omega(s)=\sup \left\{\frac{d^{N}(u(y), u(x))}{2}: \frac{d^{M}(x, y)}{2} \leq s\right\} .
$$

We prove first that if $N$ is a Hadamard space (i.e. complete and simply connected with non-positive sectional curvatures), then the modulus of continuity $\omega(., t)$ of $u(., t)$ satisfies the same inequality (9) as in the scalar 
case, depending only on a lower bound $(n-1) K$ for the Ricci curvature of $M$. The starting point is the analogue of (10), which in this setting is

$$
d^{N}(u(\gamma(1), t), u(\gamma(0), t))-2 \varphi\left(\frac{L[\gamma]}{2}, t\right) \leq 0
$$

for all smooth curves $\gamma$ in $M$ (with length close to $2 s_{0}$ ) and all $t \leq t_{0}$ close to $t_{0}$. The important thing to observe is that $d^{N}$ is smooth and convex on $\{(p, q) \in N \times N: p \neq q\}$. Thus when we carry out the same variations as in the scalar case, we obtain

$$
\begin{aligned}
0 \geq & -2 \varphi^{\prime \prime}+D d^{N}\left(u_{n n}\left(y_{0}, t_{0}\right), u_{n n}\left(x_{0}, t_{0}\right)\right) \\
& +D^{2} d^{N}\left(\left(u_{n}\left(y_{0}, t_{0}\right),-u_{n}\left(x_{0}, t_{0}\right)\right),\left(u_{n}\left(y_{0}, t_{0}\right),-u_{n}\left(x_{0}, t_{0}\right)\right)\right.
\end{aligned}
$$

in analogy with (13), and

$$
\begin{aligned}
0 \geq & -2(n-1) T_{K}\left(\frac{L}{2}\right) \varphi^{\prime}+\sum_{i<n} D d^{N}\left(u_{i i}\left(y_{0}, t_{0}\right), u_{i i}\left(x_{0}, t_{0}\right)\right) \\
& +\sum_{i<n} D^{2} d^{N}\left(\left(u_{i}\left(y_{0}, t_{0}\right),-u_{i}\left(x_{0}, t_{0}\right)\right),\left(u_{i}\left(y_{0}, t_{0}\right),-u_{i}\left(x_{0}, t_{0}\right)\right)\right.
\end{aligned}
$$

in analogy with (15). The convexity of $d^{N}$ allows us to discard the last term in both (20) and (21), and we arrive at the following in analogy with (16):

$$
D d^{N}\left(\left.\mathcal{L}[u]\right|_{\left(y_{0}, t_{0}\right)},\left.\mathcal{L}[u]\right|_{\left(x_{0}, t_{0}\right)}\right)-2 \varphi^{\prime \prime}-2(n-1) T_{K} \varphi^{\prime} \leq 0 .
$$

The time derivative inequality yields

$$
D d^{N}\left(\left.\mathcal{L}[u]\right|_{\left(y_{0}, t_{0}\right)},\left.\mathcal{L}[u]\right|_{\left(x_{0}, t_{0}\right)}\right)-2 \varphi_{t} \geq 0
$$

Combining these gives exactly the same inequality (18) as in the scalar case, proving that the modulus of continuity satisfies (9) just as in the scalar case (in this case $a=b=1$ ), and in particular we deduce a gradient bound for positive times depending only on the initial oscillation (that is, the diameter of the image set in $N$ ) and the lower bound on Ricci curvature.

Now consider the general case where $N$ is a manifold of non-positive sectional curvature, possibly with topology. In this case the distance function on $N$ is no longer smooth or convex at cut points, so the argument fails. However, we can get around this difficulty as follows: The map $u: M \times$ $[0, T) \rightarrow N$ lifts to a map $\tilde{u}$ from the universal cover $\tilde{M}$ of $M$ to the universal cover $\tilde{N}$ of $N$, which is a Hadamard space, and $\tilde{u}$ evolves by the harmonic map heat flow. Although the manifold $\tilde{M}$ may be non-compact, the map $\tilde{u}$ is co-compact, in the sense that performing deck transformation on $\tilde{M}$ simply amount to performing the corresponding deck transformations on $\tilde{N}$. The modulus of continuity $\tilde{\omega}$ of $\tilde{u}$ can therefore still be defined, and the supremum is attained at some pair of points $x_{0}$ and $y_{0}$ in $\tilde{M}$ with $x_{0}$ in some (compact) fundamental domain in $\tilde{N}$. Exactly the same argument as before shows that $\tilde{\omega}$ satisfies the inequality (9) in the viscosity sense. 
The difficulty now becomes that since $\tilde{M}$ may be non-compact, there can be issues with applying the maximum principle to prove the modulus of continuity estimate: To show $\tilde{\omega}$ stays less than some barrier $\varphi$ we need to argue that the inequality is satisfied for large values of $s$, in order to guarantee that there is some finite value of $s$ where the inequality is first violated. This can be accomplished using a nice argument from coarse geometry: Using the Švarc-Milnor theorem $[66,73]$ we prove that for each homotopy class $\mathcal{S}$ of maps in $C(M, N)$ there exists a constant $C(\mathcal{S})$ such that for every $u \in \mathcal{S}$ the lifting $\tilde{u}$ has modulus of continuity $\tilde{\omega}$ satisfying $\tilde{\omega}(s) \leq C_{1}+C(\mathcal{S}) s$ for all $s \geq 0$, where $C_{1}$ can depend on $u$. This is sufficient for our purposes: If $\varphi$ is a strict supersolution of (9) for which $\liminf _{s \rightarrow \infty} \frac{\varphi(s, t)}{s}>C(\mathcal{S})$, then automatically $\tilde{\omega}(s, t)<\varphi(s, t)$ for $s$ sufficiently large, so if the inequality is ever violated there is some finite value of $s$ where this occurs, and we derive a contradiction from the fact that $\tilde{\omega}$ is a viscosity sub solution of the same equation. In particular we can write down an explicit supersolution which still has bounded gradient for positive times, and the energy density bound follows.

The argument provides a uniform energy density bound for harmonic map heat flow which depends only on the initial modulus of continuity and the homotopy class of the map, and we deduce existence of a unique smooth solution of harmonic map heat flow starting from any continuous initial data, which exists for all time and converges to a harmonic map (some assumptions are required as usual if $N$ is non-compact to prevent the map 'escaping' to infinity).

\section{Heat flows on Ricci flows}

It has been known for a long time that the heat flow on a manifold evolving by Ricci flow has some special properties: For example, Hamilton's treatment of the short-time existence of Ricci flow using De Turck's trick of combining Ricci flow with harmonic map flow in [50, p. 20] includes the observation that in the evolution equation for energy density, the terms involving background curvature on the source manifold cancel with the terms arising from the evolution of the metric by Ricci curvature, resulting in an evolution equation which looks the same as harmonic map flow from a flat metric. Of course this applies in particular for solution of the scalar heat equation from a manifold with metric evolving by Ricci flow, so that the following evolution equation holds:

$$
\frac{\partial}{\partial t}|D u|^{2}=\Delta|D u|^{2}-2\left|D^{2} u\right|^{2}
$$

so one can for example prove gradient bounds for positive times by computing

$$
\frac{\partial}{\partial t}\left(u^{2}+2 t|D u|^{2}\right)=\Delta\left(u^{2}+2 t|D u|^{2}\right)-2 t\left|D^{2} u\right|^{2},
$$


so that $|D u|^{2} \leq \frac{\sup _{t=0} u^{2}}{2 t}$. Various other authors have observed that the interaction of the heat equation and Ricci flow leads to good gradient estimates, see $[24,65,78,79]$. The modulus of continuity argument provides a nice explanation for this phenomenon, by relating it to a special property of the distance function under Ricci flow, also first observed by Hamilton $[50,51]$. More generally, the argument works if the metric evolves as a supersolution to Ricci flow, so that $\frac{\partial g}{\partial t} \geq-2$ Rc. This includes as special cases time-independent metrics with non-negative Ricci curvature, as well as solutions of Ricci flow. This evolutionary condition seems to be the natural generalisation of non-negative Ricci curvature bounds for the timedependent context. The result on moduli of continuity (proved with David Shellard and Julie Clutterbuck [12]) is the following:

TheOREM 4. Let $M^{n}$ be a compact manifold, and $g(t)$ a time-dependent metric on $M$ satisfying $\frac{\partial g}{\partial t} \geq-2 \mathrm{Rc}$, and let $u: M \times[0, T) \rightarrow \mathbb{R}$ be a solution of the heat equation $u_{t}=\Delta u$. Then the modulus of continuity $\omega$ of $u$ satisfies $\omega_{t} \leq \omega^{\prime \prime}$ in the viscosity sense.

Note that the conclusion is just the inequality (9) for the heat equation in the case $K=0$. It remains an interesting question whether there is a corresponding evolutionary analogue of Ricci curvature lower bounds for other $K$ in the Riemannian setting: A simple inequality $\partial_{t} g \geq-2 \mathrm{Rc}+2(n-$ 1) $K g$ does not seem to suffice (but see the end of this section for a slightly more general context where a sharp result holds).

The statement also holds for harmonic map heat flow from such an evolving metric into a manifold of non-positive sectional curvature. Here we sketch the proof for the scalar case, indicating where the time-dependence of the metric enters the computation:

Proof. As before, we consider a smooth function $\varphi$ which lies above the modulus of continuity $\omega$ and is equal at some point $\left(s_{0}, t_{0}\right)$, and this gives an inequality analogous to (10):

$$
u(\gamma(1), t)-u(\gamma(0), t)-2 \varphi\left(\frac{L[\gamma, t]}{2}, t\right) \leq 0,
$$

the only difference being that the length of the curve depends explicitly on $t$ through the time-dependence of the metric $g$. The variations at fixed $t$ are therefore unchanged, yielding the first derivative identity $\nabla u\left(x_{0}, t_{0}\right)=$ $\varphi^{\prime} T(0)$ and $\nabla u\left(y_{0}, t_{0}\right)=\varphi^{\prime} T(1)$ and the inequalities (13) and (15) hold without change, but we can no longer discard the last term in (15) since we do not assume any Ricci curvature lower bound. This gives the following analogue of (16) (noting that $C_{K}(s)=1$ for $K=0$ ):

$$
\left.\mathcal{L}[u]\right|_{\left(y_{0}, t_{0}\right)}-\left.\mathcal{L}[u]\right|_{\left(x_{0}, t_{0}\right)}-2 \varphi^{\prime \prime}+\varphi^{\prime} \int_{-L / 2}^{L / 2} \operatorname{Rc}(T, T) d x \leq 0 .
$$


On the other hand, the time derivative inequality becomes

$$
\left.\mathcal{L}[u]\right|_{\left(y_{0}, t_{0}\right)}-\left.\mathcal{L}[u]\right|_{\left(x_{0}, t_{0}\right)}-2 \varphi_{t}+\varphi^{\prime} \int_{L / 2}^{L / 2} \operatorname{Rc}(T, T) d x \geq 0 .
$$

As before, the inequalities (25) and (26) combine to give

$$
\varphi_{t} \leq \varphi^{\prime \prime}
$$

completing the proof.

As noted before, this 'evolutionary' analogue of non-negative Ricci curvature does not extend easily to other lower bounds on Ricci curvature for Laplacian-type operators. However, for the drift Laplacian in BakryÉmery geometry there is a natural extension. The argument to control the modulus of continuity (and consequent bounds on eigenvalues) in the case of a time-independent Bakry-Émery setting was provided in [19].

The evolutionary analogue proved in [12] is as follows:

TheORem 5. Let $M^{n}$ be a compact manifold with time-dependent Riemannian metric $g(t)$ and smooth (time-dependent) function $f(., t)$ on $M$. Suppose that $\frac{\partial g}{\partial t} \geq-2(\operatorname{Rc}+\operatorname{Hess}(f))+2 \lambda g$ for some $a \in \mathbb{R}$. Let $u$ : $M \times[0, T) \rightarrow \mathbb{R}$ be a smooth solution of the drift-Laplacian heat flow

$$
\frac{\partial u}{\partial t}=\Delta u-g(\nabla f, \nabla u) \text {. }
$$

Then the modulus of continuity $\omega(s, t)$ of u satisfies $\partial_{t} \omega \leq \omega^{\prime \prime}-\lambda s \omega^{\prime}$ in the viscosity sense.

We note that the result is sharp, and the condition includes the 'Riemannian' condition $\partial_{t} g \geq-2 \mathrm{Rc}+2(n-1) K g$, but nevertheless the conclusion is presumably not sharp in the Riemannian setting since the equality case corresponds to a 'non-Riemannian' one-dimensional heat equation with drift - in particular, the result does not recapture the sharp result of Proposition 3 for a given lower Ricci curvature bound in the time-independent case.

ProOF. As in the proof of Proposition 3, if $\varphi$ is a smooth function which lies above $\omega$ with equality at $\left(s_{0}, t_{0}\right)$, then $(24)$ holds, with equality for $\gamma$ a length-minimising geodesic between to points $x_{0}$ and $y_{0}$ at time $t_{0}$. The first derivative identities $\nabla u\left(x_{0}, t_{0}\right)=\varphi^{\prime} T(0)$ and $\nabla u\left(y_{0}, t_{0}\right)=\varphi^{\prime} T(1)$ and the inequality (13) are unchanged, but the optimal variations for $i<n$ are now given by choosing $\gamma_{r}=e_{i}$, a parallel vector field along $\gamma_{0}$. This gives the inequality

$$
u_{i i}\left(y_{0}, t_{0}\right)-u_{i i}\left(x_{0}, t_{0}\right)+\varphi^{\prime} \int_{\gamma_{0}} R\left(e_{i}, T, e_{i}, T\right) d s \leq 0
$$

for each $i<n$. Combining these yields the following:

$$
\left.\Delta u\right|_{\left(y_{0}, t_{0}\right)}-\left.\Delta u\right|_{\left(x_{0}, t_{0}\right)}-2 \varphi^{\prime \prime}+\varphi^{\prime} \int_{\gamma_{0}} \operatorname{Rc}(T, T) d s \leq 0 .
$$


The time derivative inequality is as follows:

$$
\begin{aligned}
0 \leq & \left.\Delta u\right|_{\left(y_{0}, t_{0}\right)}-\left.\nabla u \cdot \nabla f\right|_{\left(y_{0}, t_{0}\right)}-\left.\Delta u\right|_{\left(x_{0}, t_{0}\right)}+\left.\nabla u \cdot \nabla f\right|_{\left(x_{0}, t_{0}\right)} \\
& -2 \varphi_{t}+\varphi^{\prime} \int_{\gamma_{0}} \operatorname{Rc}(T, T)+\operatorname{Hess}(f)(T, T)-\lambda g(T, T) d s \\
\leq & \left.\Delta u\right|_{\left(y_{0}, t_{0}\right)}-\left.\varphi^{\prime} T \cdot \nabla f\right|_{\gamma_{0}(1)}-\left.\Delta u\right|_{\left(x_{0}, t_{0}\right)}+\left.\varphi^{\prime} T \cdot \nabla f\right|_{\gamma_{0}(0)} \\
& -2 \varphi_{t}+\varphi^{\prime} \int_{\gamma_{0}} \operatorname{Rc}(T, T) d s+\left.\varphi^{\prime} \nabla f \cdot T\right|_{0} ^{1}-\lambda \varphi^{\prime} L \\
= & \left.\Delta u\right|_{\left(y_{0}, t_{0}\right)}-\left.\Delta u\right|_{\left(x_{0}, t_{0}\right)}-2 \varphi_{t}-\lambda \varphi^{\prime} L-\varphi^{\prime} \int_{\gamma_{0}} \operatorname{Rc}(T, T) d s .
\end{aligned}
$$

Combining the two inequalities gives $\varphi_{t}(x, t) \leq \varphi^{\prime \prime}(x, t)-\lambda x \varphi^{\prime}(x, t)$, as required.

\section{Height-dependent gradient bounds}

A related approach to the modulus of continuity argument allows us to prove a more sophisticated gradient estimate, although for this we can only deal with the heat equation and a much smaller class of nonlinear parabolic equations. The idea here is to derive the sharp bound on the gradient of a solution, as a function of the value at that point. Motivated by the gradient estimates we inferred from the modulus of continuity estimate, here we derive height-dependent gradient estimates by considering a generalised modulus of continuity which depends on the height. We phrase the result as a comparison between an arbitrary solution and a 'one-dimensional' solution, and for simplicity we consider only the case of non-negative Ricci curvature:

THEOREM 6. Let $\left(M^{n}, g\right)$ be a compact Riemannian manifold with diameter $D$ and Ricci curvature satisfying $\mathrm{Rc} \geq 0$, and suppose $u: M \times$ $[0, T) \rightarrow \mathbb{R}$ is a smooth solution of an equation of the form

$$
\frac{\partial u}{\partial t}=\left[a(u,|D u|) \frac{u_{i} u_{j}}{|D u|^{2}}+b\left(g^{i j}-\frac{u_{i} u_{j}}{|D u|^{2}}\right)\right] u_{i j},
$$

with $b$ constant. Let $\varphi:[0, D] \times[0, T) \rightarrow \mathbb{R}$ be a solution of $\varphi_{t}=a\left(\varphi, \varphi^{\prime}\right) \varphi^{\prime \prime}$ with Neumann boundary condition, which is increasing in the first argument, such that the range of $u(., 0)$ is contained in the interval $[\varphi(0,0), \varphi(D, 0)]$. Let $\Psi$ be given by inverting $\varphi$ for each $t$, so that $\varphi(\Psi(z, t), t)=z$ for each $z$ and $t$, and assume that for all $x$ and $y$ in $M$,

$$
\Psi(u(y, 0), 0)-\Psi(u(x, 0), 0)-d^{M}(x, y) \leq 0 .
$$

Then

$$
\Psi(u(y, t), t)-\Psi(u(x, t), t)-d^{M}(x, y) \leq 0
$$

for all $x, y \in M$ and $t \in[0, T)$. 
While the condition that $b$ is constant is quite restrictive (ruling out important examples such as $p$-Laplacian heat flows), there are some interesting examples in this class other than the heat equation itself: The graphical mean curvature flow is an important example, and another is (formally) the $\infty$-Laplacian heat flow.

By allowing $y$ to approach $x$, we deduce the following sharp gradient bound as a function of the height:

Corollary 7. Under the conditions of Theorem 6, for every $x \in M$ and $t \geq 0$ we have

$$
|\nabla u(x, t)| \leq \varphi^{\prime}(\Psi(u(x, t), t), t) .
$$

In fact it is straightforward to see that Corollary 7 is equivalent to Theorem 6 . The result of Corollary 7 is closely related to results proved for eigenfunctions using maximum principle estimates applied to the gradient, as in $[25,60]$. As can be seen below, the argument via Theorem 6 and multipoint maximum principles is much easier and more direct than the argument using gradient estimates, and provides a nice connection with Laplacian comparison principles.

Proof of Theorem 6 . Let $\varepsilon>0$ be arbitrary, and consider the first time $t_{0}>0$ and points $x_{0}$ and $y_{0}$ in $M$ at which the inequality

$$
\Psi(u(y, t), t)-\Psi(u(x, t), t)-d^{M}(x, y)-\varepsilon(1+t) \leq 0
$$

reaches equality. Note that if $\varepsilon>0$ then we necessarily have $y_{0} \neq x_{0}$. Then we also have

$$
\Psi(u(\gamma(1), t), t)-\Psi(u(\gamma(0), t), t)-L[\gamma]-\varepsilon(1+t) \leq 0
$$

for all smooth curves $\gamma$ in $M$, with equality when $t=t_{0}$ and $\gamma=\gamma_{0}$, a length-minimising geodesic from $x_{0}$ to $y_{0}$. Varying the endpoints gives the first order identities

$$
\Psi^{\prime}\left(u\left(y_{0}, t_{0}\right), t_{0}\right) \nabla u\left(y_{0}, t_{0}\right)-T(1)=0
$$

and

$$
\Psi^{\prime}\left(u\left(x_{0}, t_{0}\right)\right)-\nabla u\left(x_{0}, t_{0}\right) \cdot T(0)=0,
$$

so that

$$
\nabla u\left(y_{0}, t_{0}\right)=\frac{1}{\Psi^{\prime}\left(y_{0}, t_{0}\right)} T(1)=\varphi^{\prime}\left(z_{y}, t_{0}\right) T(1)
$$

and

$$
\nabla u\left(y_{0}, t_{0}\right)=\frac{1}{\Psi^{\prime}\left(y_{0}, t_{0}\right)} T(1)=\varphi^{\prime}\left(z_{x}, t_{0}\right) T(0)
$$

where $z_{y}=\Psi\left(u\left(y_{0}, t_{0}\right)\right)$ and $z_{x}=\Psi\left(u\left(x_{0}, t_{0}\right)\right)$ represent the arguments for which $\varphi\left(., t_{0}\right)$ has the same height as $u\left(., t_{0}\right)$ at the points $y_{0}$ and $x_{0}$ respectively, and $T$ is the unit tangent vector to $\gamma_{0}$. Next we compute the second derivatives, under suitable variations. Note that in the model one-dimensional case, equality holds at all points of the form 
$\left(X\left(z, s_{1}, t\right), X\left(z, s_{2}, t\right)\right)$ for any $z \in \Sigma_{h}, s_{1}, s_{2} \in J$ and $\left.t \in 0, T\right)$. Thus we have more allowed directions of variation than we had for the modulus of continuity argument, since we can move $x_{0}$ and $y_{0}$ independently along the curve $\gamma_{0}$. Moving $y_{0}$ gives the inequality

$$
\begin{gathered}
0 \geq \frac{u_{n n}\left(y_{0}, t_{0}\right)}{\varphi^{\prime}\left(z_{y}\right)}-\frac{u_{n}\left(y_{0}, t_{0}\right)^{2}}{\varphi^{\prime}\left(z_{y}\right)^{3}} \varphi^{\prime \prime}\left(z_{y}\right) \\
=\frac{u_{n n}\left(y_{0}, t_{0}\right)-\varphi^{\prime \prime}\left(z_{y}\right)}{\varphi^{\prime}\left(z_{y}\right)} .
\end{gathered}
$$

Similarly we have by moving $x_{0}$ along $\gamma_{0}$

$$
0 \geq-\frac{u_{n n}\left(x_{0}, t_{0}\right)-\varphi^{\prime \prime}\left(z_{x}\right)}{\varphi^{\prime}\left(z_{x}\right)}
$$

In the transverse directions we choose as before the variation corresponding to a parallel vector field $e_{i}$, yielding the following inequality:

$$
\frac{u_{i i}\left(y_{0}, t_{0}\right)}{\varphi^{\prime}\left(z_{y}\right)}-\frac{u_{i i}\left(x_{0}, t_{0}\right)}{\varphi^{\prime}\left(z_{x}\right)}+\int_{\gamma_{0}} R\left(e_{i}, T, e_{i}, T\right) \leq 0 .
$$

Adding these over $i<n$ and using the nonnegative Ricci curvature condition gives

$$
\sum_{i<n} \frac{u_{i i}\left(y_{0}, t_{0}\right)}{\varphi^{\prime}\left(z_{y}\right)}-\sum_{i<n} \frac{u_{i i}\left(x_{0}, t_{0}\right)}{\varphi^{\prime}\left(z_{x}\right)} \leq 0 .
$$

Now the time-derivative inequality gives

$$
\begin{aligned}
\varepsilon \leq & \left.\left(\Psi_{t}+\Psi^{\prime} u_{t}\right)\right|_{\left(y_{0}, t_{0}\right)}-\left.\left(\Psi_{t}+\Psi^{\prime} u_{t}\right)\right|_{\left(x_{0}, t_{0}\right)} \\
= & \left.\left(-\frac{\varphi_{t}}{\varphi^{\prime}}+\frac{u_{t}}{\varphi^{\prime}}\right)\right|_{\left(y_{0}, t_{0}\right)}-\left.\left(-\frac{\varphi_{t}}{\varphi^{\prime}}+\frac{u_{t}}{\varphi^{\prime}}\right)\right|_{\left(x_{0}, t_{0}\right)} \\
= & \frac{1}{\varphi^{\prime}\left(z_{y}\right)}\left(-a\left(\varphi\left(z_{y}\right), \varphi^{\prime}\left(z_{y}\right)\right) \varphi^{\prime \prime}+a\left(\varphi\left(z_{y}\right), \varphi^{\prime}\left(z_{y}\right)\right) u_{n n}\left(y_{0}, t_{0}\right)+b \sum_{i<n} u_{i i}\left(y_{0}, t_{0}\right)\right) \\
& -\frac{1}{\varphi^{\prime}\left(z_{x}\right)}\left(-a\left(\varphi\left(z_{x}\right), \varphi^{\prime}\left(z_{x}\right)\right) \varphi^{\prime \prime}+a\left(\varphi\left(z_{x}\right), \varphi^{\prime}\left(z_{x}\right)\right) u_{n n}\left(x_{0}, t_{0}\right)+b \sum_{i<n} u_{i i}\left(x_{0}, t_{0}\right)\right) \\
(41)) & \frac{a\left(\varphi\left(z_{y}\right), \varphi^{\prime}\left(z_{y}\right)\right)}{\varphi^{\prime}\left(z_{y}\right)}\left(u_{n n}\left(y_{0}, t_{0}\right)-\varphi^{\prime \prime}\left(z_{y}\right)\right)+\frac{a\left(\varphi\left(z_{x}\right), \varphi^{\prime}\left(z_{x}\right)\right)}{\varphi^{\prime}\left(z_{x}\right)}\left(\varphi^{\prime \prime}\left(z_{x}\right)-u_{n n}\left(x_{0}, t_{0}\right)\right) \\
& +b\left(\sum_{i<n} \frac{u_{i i}\left(y_{0}, t_{0}\right)}{\varphi^{\prime}\left(z_{y}\right)}-\sum_{i<n} \frac{u_{i i}\left(x_{0}, t_{0}\right)}{\varphi^{\prime}\left(z_{x}\right)}\right) \\
\leq & 0,
\end{aligned}
$$

a contradiction. Here we used the inequalities (37),(38) and (39) in the three terms in the last line. We conclude that the inequality (34) is never violated for any $\varepsilon>0$, proving (33).

Examples of gradient bounds which follow immediately from this include the following: 
(1) If $u$ is a bounded solution of the heat equation on Euclidean space with $|u(x, 0)| \leq M$ for all $x$, then $|\nabla u(x, t)|$ is no greater than the gradient of the function $M \operatorname{erf}\left(\frac{x}{2 \sqrt{t}}\right)$ at the point with height $u(x, t)$ :

$$
|\nabla u(x, t)| \leq \frac{M}{\sqrt{\pi t}} \mathrm{e}^{-\left(\operatorname{erf}^{-1}\left(\frac{u(x, t)}{M}\right)\right)^{2}} .
$$

The inequality $\mathrm{e}^{-\left(\operatorname{erf}^{-1}(z)\right)^{2}} \leq 4 \sqrt{\pi}(1-z)$ holds for $z>0$, so we can deduce the simpler (but non-sharp) expression $|\nabla u| \leq$ $\frac{4}{\sqrt{t}}(M-\mid u(x, t \mid)$.

(2) If $u$ is a non-positive solution of the graphical mean curvature flow, then the gradient at given height is bounded by that of the solution of graphical curvature shortening flow expanding from the boundary of the quadrant $\{x>0, y<0\}$, which has the form $\varphi(x, t)=\sqrt{t} F(x / \sqrt{t})$ where $F$ satisfies the ODE $F^{\prime \prime}(z)=$ $\frac{1}{2}\left(F-F^{\prime} z\right)\left(1+\left(F^{\prime}\right)^{2}\right)$ with $F \rightarrow-\infty$ as $z \rightarrow 0$ and $F \rightarrow 0$ as $z \rightarrow \infty$. This captures a sharp form of the gradient estimate of Evans and Spruck [44, Theorem 5.2].

(3) If $u$ is a solution of the heat equation on hyperbolic space, then the gradient at a given height is bounded by that of the one-dimensional solution starting from Heaviside initial data. In particular, if $u$ is a harmonic function with $|u| \leq M$ on $\mathbb{H}^{n}$, then the estimate on the gradient is given by taking the large time limit. With some extra work this estimate can be localised, and this gives a sharp form of Yau's gradient estimate from [75], with equality (in the limit of large radius) given by functions of the form $u=C_{1}+C_{2} F_{n}(s)$, where $s$ is the geodesic distance to a totally geodesic hypersurface in $\mathbb{H}^{n}$ and $F_{n}(s)=\int_{0}^{s} \frac{d \sigma}{(\cosh \sigma)^{n-1}}$ (given by solving the Dirichlet problem at infinity with boundary data equal to different constants on two hemispheres of the sphere at infinity).

For the heat equation (with $a=b=1$ ), we also have results corresponding to Theorem 6 and Corollary 7 for time-dependent metrics satisfying $\frac{\partial}{\partial t} g \geq-2$ Rc (reproducing and sharpening several of the results in $[24,65,78,79]$, and corresponding results hold for the drift-Laplacian in a possibly time-dependent Bakry-Émery setting [12].

\section{Nonlinear eigenvalue equations}

We discussed previously the application of modulus of continuity estimates to eigenvalue estimates. Since the modulus of continuity estimates are effective for a range of nonlinear parabolic equations [9], there are also useful applications to a variety of nonlinear eigenvalue problems, which we treat in [13]. The best-known examples of these are the $p$-Laplacian eigenvalues, which are solutions of the equation

$$
0=\Delta_{p} u+\lambda|u|^{p-2} u=D_{i}\left(|D u|^{p-2} D_{i} u\right)+\lambda^{p-1}|u|^{p-2} u,
$$


which arise from minimising the Rayleigh quotient

$$
\mathcal{E}_{p}[u]=\int|D u|^{p}
$$

subject to the constraint $\int|u|^{p-2} u=0$.

We emphasise that the solutions of (43) do not interact naturally with the $p$-Laplacian heat flow, and instead it is natural to consider a more nonlinear version of the heat flow associated to the $p$-Laplacian: The usual $p$-Laplacian heat equation corresponds to taking the $L^{2}$ gradient flow of the energy $\mathcal{E}_{p}$. However, if we take the steepest descent flow in $L^{p}$ for the energy $\mathcal{E}_{p}$, we are led to the following fully nonlinear flow:

$$
\frac{\partial u}{\partial t}=\left|\Delta_{p} u\right|^{-\frac{p-2}{p-1}} \Delta_{p} u \text {. }
$$

We leave aside questions of existence and regularity for this flow, but merely observe that the $p$-eigenfunctions lead to similarity solutions which decay exponentially at rate $\mathrm{e}^{-\lambda t}$. Thus an exponential rate of decay on the oscillation of solutions implies a lower bound on the first nontrivial eigenvalue $\lambda$, and we can achieve this using the modulus of continuity argument with some modifications, but only in the case $1<p \leq 2-$ we will discuss how to handle the case $p>2$ by different methods below.

The modulus of continuity argument begins exactly as in the previous cases: If $u$ is a solution of (44) on $M$ with modulus of continuity $\omega(s, t)$, and $\varphi$ is smooth and lies above $\omega$ with equality at $\left(s_{0}, t_{0}\right)$, then we have the inequality (10) for all curves $\gamma$, with equality for $\gamma=\gamma_{0}$, a minimising geodesic from $x_{0}$ to $y_{0}$ of length $2 s_{0}$. Exactly as before we have $\nabla u\left(x_{0}, t_{0}\right)=\varphi^{\prime} T(0)$ and $\nabla u\left(y_{0}, t_{0}\right) T(1)$, and exactly the same second derivative inequalities (13) and (15) hold. In this case we have $a\left(\varphi^{\prime}\right)=(p-1)\left|\varphi^{\prime}\right|^{p-2}$ and $b\left(\varphi^{\prime}\right)=\left|\varphi^{\prime}\right|^{p-2}$, so (16) becomes

$$
\Delta_{p} u\left(y_{0}, t_{0}\right)-\Delta_{p} u\left(x_{0}, t_{0}\right)-2 \mathcal{L}_{p, K}[\varphi] \leq 0,
$$

where $\mathcal{L}_{p, K}[\varphi]=(p-1)\left|\varphi^{\prime}\right|^{p-2} \varphi^{\prime \prime}+(n-1) T_{K}\left|\varphi^{\prime}\right|^{p-2} \varphi^{\prime}$. However the time derivative inequality becomes the following:

$$
\left.\left|\Delta_{p} u\right|^{-\frac{p-2}{p-1}} \Delta_{p} u\right|_{\left(y_{0}, t_{0}\right)}-\left.\left|\Delta_{p} u\right|^{-\frac{p-2}{p-1}} \Delta_{p} u\right|_{\left(x_{0}, t_{0}\right)}-2 \varphi_{t} \geq 0 .
$$

In the case $1<p \leq 2$ we observe that $f(z)=|z|^{-\frac{p-2}{p-1}} z$ is an increasing odd function which is convex for positive $z$, and deduce the inequality $f(z-2 \delta)-f(z) \leq-2 f(\delta)$ for every $z \in \mathbb{R}$ and $\delta \geq 0$. Choosing $z=$ $\Delta_{p} u\left(x_{0}, t_{0}\right)$ and $\delta=-\mathcal{L}[\varphi]$, the inequality (45) gives $\Delta_{p} u\left(y_{0}, t_{0}\right) \leq z-2 \delta$, and hence $f\left(\Delta_{p} u\left(y_{0}, t_{0}\right)\right)-f\left(\Delta_{p} u\left(x_{0}, t_{0}\right)\right) \leq f(z-2 \delta)-f(z) \leq-2 f(\delta)$, so that

$$
\left.\left|\Delta_{p} u\right|^{-\frac{p-2}{p-1}} \Delta_{p} u\right|_{\left(y_{0}, t_{0}\right)}-\left.\left|\Delta_{p} u\right|^{-\frac{p-2}{p-1}} \Delta_{p} u\right|_{\left(x_{0}, t_{0}\right)}-2 f\left(\mathcal{L}_{p, K}[\varphi]\right) \leq 0 .
$$

Finally, the two inequalities (46) and (47) yield the inequality

$$
\varphi_{t} \leq f\left(\mathcal{L}_{p, K}[\varphi]\right)
$$


which says precisely that $\omega$ is a viscosity subsolution of the one-dimensional model equation corresponding to (44). Finally, we can now use the eigenfunction corresponding to the model situation as a barrier to show that arbitrary solutions of (44) decay exponentially, proving that the first nontrivial $p$-eigenvalue is no smaller than the eigenvalue for the corresponding one-dimensional equation on an interval of the same diameter. This gives the sharp lower bound on the first eigenvalue for this range of $p$, for any given diameter and lower Ricci curvature bound. The result for non-negative Ricci curvature was proved previously (for any $p>1$ ) by Valtorta [74], and the special case of convex domains in Euclidean space was proved independently in [43]. The result for arbitrary $K$ was proved by Valtorta and Naber [67].

The methods outlined above can also be applied to a range of fully nonlinear equations satisfying certain concavity assumptions. However, there seems to be no way to make the argument work for $p>2$. Nevertheless, the optimal lower bound for $p$-eigenvalues as a function of diameter and lower Ricci curvature bound for $p>2$ can be proved using methods similar to the height-dependent gradient estimates discussed above. In this case, however, we apply the estimates not to the evolution equation but instead to the eigenfunction equation, and the argument is therefore much closer to that originally used in the works of $[25,60,63,64]$ for $p=2$ and by Valtorta in [74] and Valtorta and Naber in [67]. Indeed the key gradient estimate we prove is essentially the same as in [67], but the argument is made much easier by the use of multi-point maximum principles rather than direct estimation of the gradient.

We will prove the basic gradient estimate for eigenfunctions of the $p$ Laplacian, by means of a generalised modulus of continuity estimate of the kind we used in section 7 .

In this setting (as in $[25,67,74])$ it is useful to expand our class of 'onedimensional' solutions to include all 'warped product' solutions in the model space $\bar{M}_{K}$ : For given $K \in \mathbb{R}$ and $h>0$, let $\Sigma_{h}$ be a hypersurface with constant principal curvatures equal to $h$ in the model space $\bar{M}_{K}$ of constant sectional curvature $K$, and let $\nu(z)$ be the outward unit normal to $\Sigma_{h}$ at $z \in \Sigma_{h}$. Define $X_{h}: \Sigma \times I_{h, K} \rightarrow \bar{M}_{K}$ given by $X_{h}(z, s)=\exp _{z}(s \nu(z))$, where $I_{h, K}$ is the maximal interval on which this map is a diffeomorphism. We say $\varphi: J \times[0, T) \rightarrow \mathbb{R}$ is a one-dimensional solution of a heat flow (43) on $\bar{M}_{K}$ if there is some $h \geq 0$ such that $J \subset I_{h, K}$ and the function $u$ defined by $u\left(X_{h}(z, s), t\right)=\varphi(s, t)$ satisfies (43). We have the following result:

TheOrem 8. Suppose that $(M, g)$ is a compact Riemannian manifold satisfying $\mathrm{Rc} \geq(n-1) K g$ for some $K \in \mathbb{R}$, and suppose that $u$ is a $p$ eigenfunction:

$$
\left[(p-1) \frac{u_{i} u_{j}}{|D u|^{2}}+|D u|^{p-2}\left(g^{i j}-\frac{u_{i} u_{j}}{|D u|^{2}}\right)\right] u_{i j}+\lambda|u|^{p-2} u=0 .
$$

Suppose $\varphi:[0, D] \times[0, T) \rightarrow \mathbb{R}$ is a one-dimensional solution of (43) which is increasing, and such that the range of $u$ is contained in $[\varphi(0), \varphi(D)]$. Let 
$\Psi$ be the inverse function of $\varphi$. Then for every $x$ and $y$ in $M$,

$$
\Psi(u(y))-\Psi(u(x))-d^{M}(x, y) \leq 0 .
$$

Proof. In the proof, we proceed as in Theorem 6, and consider the smallest $c$ such that

$$
Z(x, y)=\Psi\left(\frac{u(y)}{c}\right)-\Psi\left(\frac{u(x)}{c}\right)-d^{M}(x, y) \leq 0,
$$

so that equality holds for some $(y, x)=\left(y_{0}, x_{0}\right)$ with $y_{0} \neq x_{0}$, and suppose ${ }^{3}$ for the purposes of obtaining a contradiction that $c>1$. This is equivalent to the situation in the proof of Theorem 6 where we have replaced $\varphi$ by $c \varphi$. Note that $c \varphi$ is again a solution of the one-dimensional eigenvalue equation, and let $\bar{u}$ be the corresponding solution of (43) on $\bar{M}$. Writing $z_{x}$ and $z_{y}$ for the points where $c \varphi\left(z_{x}\right)=u(x)$ and $c \varphi\left(z_{y}\right)=u(y)$, the first derivative identities give $\nabla u(y)=c \varphi^{\prime}\left(z_{y}\right) T$ and $\nabla u(x)=c \varphi^{\prime}\left(z_{y}\right) T$, where $T$ is the unit tangent vector to a minimising geodesic $\gamma_{0}$ from $x_{0}$ to $y_{0}$. As in (37) and (38), the second variations in $x$ and $y$ along $\gamma_{0}$ also produce the identities

$$
\left(\frac{\partial}{\partial x^{n}}\right)^{2} Z=\frac{1}{c \varphi^{\prime}\left(z_{x}\right)}\left(c \varphi^{\prime \prime}\left(z_{x}\right)-u_{n n}(x)\right)
$$

and

$$
\left(\frac{\partial}{\partial y^{n}}\right)^{2} Z=\frac{1}{c \varphi^{\prime}\left(z_{y}\right)}\left(u_{n n}(y)-c \varphi^{\prime \prime}\left(z_{y}\right)\right) \leq 0 .
$$

In the transverse directions, we must choose a variation for which equality holds in the one-dimensional solution: That is, we define $\bar{f}$ satisfying $\bar{f}^{\prime \prime}+$ $K \bar{f}=0$ on $I_{h, K}$ with $\bar{f}(0)=1$ and $\bar{f}^{\prime}(0)=h$, corresponding to the variation $\gamma(r, s)=X_{h}(z(r), s)$ in the model space. Then we set $\gamma_{r}(s)=\bar{f}\left((1-s) z_{x}+\right.$ $\left.s z_{y}\right) \bar{e}$ where $\bar{e}$ is parallel along $\gamma_{0}$ and $s$ is the arc length parameter. Since the lower bound on Ricci curvature guarantees an inequality for the second variation of lengths, we obtain (from the fact that $Z$ is identically zero under this variation in the model case) the identity

$$
\begin{aligned}
& \sum_{i<n}\left(\bar{f}\left(z_{y}\right) \frac{\partial}{\partial y^{i}}+\bar{f}\left(z_{x}\right) \frac{\partial}{\partial x^{i}}\right)^{2} Z \\
& \quad \geq \sum_{i<n}\left(\bar{f}\left(z_{y}\right)^{2}\left(u_{i i}(y)-\bar{u}_{i i}\left(z_{y}\right)\right)+\bar{f}\left(z_{x}\right)^{2}\left(\bar{u}_{i i}\left(z_{x}\right)-u_{i i}(x)\right)\right) .
\end{aligned}
$$

\footnotetext{
${ }^{3}$ See [13] for a more careful treatment including discussion of why such $c, x_{0}$ and $y_{0}$ exist and why the possible lack of regularity of $p$-eigenfunctions is not an obstacle to the argument.
} 
Combining this with the inequalities (51) and (52), we obtain the following inequality:

$$
\begin{aligned}
& (p-1)\left|D \bar{u}\left(z_{x}\right)\right| \bar{f}\left(z_{x}\right)^{2}\left(\frac{\partial}{\partial x^{n}}\right)^{2} Z+(p-1)\left|D \bar{u}\left(z_{y}\right)\right| \bar{f}\left(z_{y}\right)^{2}\left(\frac{\partial}{\partial y^{n}}\right)^{2} Z \\
& +\sum_{i<n}\left(\bar{f}\left(z_{y}\right) \frac{\partial}{\partial y^{i}}+\bar{f}\left(z_{x}\right) \frac{\partial}{\partial x^{i}}\right)^{2} Z \\
& \geq \frac{\bar{f}\left(z_{y}\right)^{2}}{\left|D \bar{u}\left(z_{y}\right)\right|^{p-2}}\left(\Delta_{p} u(y)-\Delta_{p} \bar{u}\left(z_{y}\right)\right)+\frac{\bar{f}\left(z_{x}\right)^{2}}{\left|D \bar{u}\left(z_{x}\right)\right|^{p-2}}\left(\Delta_{p} \bar{u}\left(z_{x}\right)-\Delta_{p} u(x)\right) \\
& =0
\end{aligned}
$$

since $\Delta_{p} u=-\lambda|u|^{p-2} u=-\lambda|\bar{u}|^{p-2} \bar{u}=\Delta_{p} \bar{u}$ at the corresponding points. We conclude from the strong maximum principle that $Z$ must be constant (and equal to zero) along the geodesic $\gamma_{0}$, so that $u\left(\gamma_{0}(s)=c \varphi\left(z_{x}+s\right)\right.$ along this curve. But this contradicts the assumption on the range of $u$, since this is a strict subset of the range of $c \varphi$ if $c>1$. Therefore the inequality holds with $c=1$ and the proof is complete.

It follows that the diameter of $M$ is no less than that of the domain of $\varphi$, and this implies that $\lambda$ is no less than the $p$-eigenvalue of the onedimensional eigenfunction with domain of the same diameter as $M$ and range the same as $u$. The optimal lower bound on the $p$-eigenvalue for given diameter then follows, since it was shown in [67] that the lowest eigenvalue for a one-dimensional $p$-eigenfunction with given diameter occurs in the case where the range is symmetric.

We remark that the argument is unchanged if the operator is replaced by any suitably homogeneous eigenfunction equation for an isotropic operator.

\section{Log-concavity and the fundamental gap}

Another successful use of multi-point estimates occurs in the proof of the fundamental gap conjecture [8]. Here the objective is to derive an optimal lower bound on the difference between the first two Dirichlet eigenvalues for the Laplacian (possibly with potential) on a convex Euclidean domain. This problem arose in the 1980s, independently conjectured by Yau [72], van den Berg [27] and Ashbaugh and Benguria [23].

A landmark early result was proved using gradient estimates in [72]. The idea here is to apply gradient estimates to the ratio $v=\frac{u_{1}}{u_{0}}$, where $u_{0}$ is the (positive) first Dirichlet eigenfunction (with eigenvalue $\lambda_{0}$ ), and $u_{1}$ is another eigenfunction (with eigenvalue $\lambda_{1}$. Then $v$ satisfies the equation

$$
\Delta v+2 \nabla \log u_{0} \cdot \nabla v+\left(\lambda_{1}-\lambda_{0}\right) v=0
$$

with Neumann boundary condition (the normal derivative of $v$ vanishes on the boundary of the domain). This is very similar to the Neumann eigenfunction equation, and similar gradient estimate (with some control on the 'drift' velocity $\nabla \log u_{0}$ ) yields a lower bound on $\lambda_{1}-\lambda_{0}$. Accordingly, 
it makes sense to try to use the modulus of continuity argument to obtain the lower bound. More precisely, given an arbitrary solution of the Dirichlet heat equation with potential,

$$
\begin{aligned}
\frac{\partial}{\partial t} u & =\Delta u-V u, \quad \text { on } \Omega \times[0, \infty) ; \\
u & =0 \quad \text { on } \partial \Omega \times[0, \infty),
\end{aligned}
$$

we let $u_{0}(x, t)=\mathrm{e}^{-\lambda_{0} t} u_{0}(x)$ where $u_{0}$ is the first eigenfunction (so that $u_{0}>0$ and $\Delta u_{0}-V u_{0}+\lambda_{0} u_{0}=0$ on $\Omega$ with $u=0$ on $\left.\partial \Omega\right)$. Then we set $v(x, t)=\frac{u(x, t)}{u_{0}(x, t)}$, and observe that $v$ satisfies the modified Neumann heat equation

$$
\begin{aligned}
\frac{\partial}{\partial t} v & =\Delta v+2 \nabla \log u_{0} \cdot \nabla v \\
D_{\nu} v & =0 \quad \text { on } \partial \Omega .
\end{aligned}
$$

An exponentially decaying bound on the oscillation of $v$ implies a lower bound on $\lambda_{1}-\lambda_{0}$. Accordingly we try to show that the modulus of continuity of $v$ is a viscosity subsolution of a natural equation arising from the onedimensional situation.

The argument proceeds exactly as in section 2 : If $\varphi$ is a smooth function lying above $\omega$, then the inequality (2) holds (with $u$ replaced by $v$ ), and the time-derivative inequality (3), the first derivative identities (4) and (5) hold, and the second derivative inequalities (7) and (8) hold (again, all with $u$ replaced by $v$ ). The difference from section 2 occurs only when we substitute the time derivative: From (55) and (3) we have

$$
\begin{aligned}
2 \frac{\partial \varphi}{\partial t} \leq & \left(\Delta v+2 \nabla \log u_{0} \cdot \nabla v||_{\left(y_{0}, t_{0}\right)}-\left(\Delta v+2 \nabla \log u_{0} \cdot \nabla v||_{\left(x_{0}, t_{0}\right)}\right.\right. \\
= & v_{n n}\left(y_{0}, t_{0}\right)-v_{n n}\left(x_{0}, t_{0}\right)+\sum_{i<n}\left(v_{i i}\left(y_{0}, t_{0}\right)-v_{i i}\left(x_{0}, t_{0}\right)\right) \\
& +2\left(\nabla \log u_{0}\left(y_{0}, t_{0}\right)-\nabla \log u_{0}\left(x_{0}, t_{0}\right)\right) \cdot \varphi^{\prime} \frac{y_{0}-x_{0}}{\left|y_{0}-x_{0}\right|} \\
\leq & 2 \varphi^{\prime \prime}+2\left(\nabla \log u_{0}\left(y_{0}, t_{0}\right)-\nabla \log u_{0}\left(x_{0}, t_{0}\right)\right) \cdot \varphi^{\prime} \frac{y_{0}-x_{0}}{\left|y_{0}-x_{0}\right|},
\end{aligned}
$$

where we used the first derivative identities (4) and (5) to produce the second line, and applied the second derivative inequalities (8) and (7) to produce the last line. To proceed we need to find a useful inequality on the final term, and clearly to get a sharp result we must estimate this term in such a way that equality holds in one-dimensional model case.

It is clear that inequalities on this term are related to log-concavity of the first eigenfunction $u_{0}$ : If $\log u_{0}$ is concave, then the last term is non-positive. In fact this is true for the first eigenfunction of the Dirichlet Laplacian with convex potential, as proved first by Brascamp and Lieb [28], and later by Korevaar [59] (using an argument originating with Yau involving a 'concavity maximum principle' involving several points of the kind described 
in this article), with later variations on the argument provided by Caffarelli and Spruck [36] and Caffarelli and Friedman [35]. A rather more direct argument is given by Yau [72, Appendix B] using a continuity argument and a strong maximum principle applied to the Hessian of $\log u_{0}$. This result means that the final term can be discarded, allowing a comparison with the one-dimensional heat equation and yields the estimate $\lambda_{1}-\lambda_{0} \geq \frac{\pi^{2}}{D^{2}}$, where $D$ is the diameter of the domain ${ }^{4}$. However, we are throwing away something nontrivial here since in the one-dimensional case $\log u_{0}$ is strictly concave.

This leads us to prove a stronger log-concavity estimate, by proving an inequality on the final term in (56). The idea is very closely analogous to that for controlling the modulus of continuity, but instead we define a modulus of convexity for the logarithm of a positive solution of the Dirichlet heat equation (possibly with potential): If $u: \Omega \times[0, \infty) \rightarrow \mathbb{R}_{+}$satisfies the heat equation with Dirichlet boundary conditions, we define

$$
\sigma(s, t)=\sup \left\{\frac{1}{2}(\nabla \log u(y, t)-\nabla \log u(x, t)) \cdot \frac{y-x}{|y-x|}:|y-x|=2 s\right\} .
$$

Thus a non-positive modulus of convexity implies that $\log u(., t)$ is concave, and a strict negativity tells us something more. We will show that $\sigma$ is a viscosity subsolution of an equation arising from the one-dimensional situation. Accordingly, suppose $\varphi$ is any smooth function which lies above $\sigma$ but is equal to $\sigma$ as some point $\left(s_{0}, t_{0}\right) \in\left(0, \frac{D}{2}\right)$, and let $x_{0}$ and $y_{0}$ be points where the supremum is attained in the definition of $\sigma\left(s_{0}, t_{0}\right)$ is attained. Then we have the inequality

$$
(\nabla \log u(y, t)-\nabla \log u(x, t)) \cdot \frac{y-x}{|y-x|} \leq 2 \varphi\left(\frac{|y-x|}{2}, t\right),
$$

and equality holds when $y=y_{0}, x=x_{0}$, and $t=t_{0}$. For convenience we write $w=\log u$ and $d=|y-x|$. We choose an orthonormal basis for $\mathbb{R}^{n}$ such that $e_{n}=\frac{y_{0}-x_{0}}{\left|y_{0}-x_{0}\right|}$. As usual, since both sides of the equation are smooth, we have first derivative identities and second derivative inequalities at the point $\left(x_{0}, y_{0}, t_{0}\right)$ : The spatial first derivative identities give

$$
w_{i n}\left(y_{0}\right)+\frac{w_{i}\left(y_{0}\right)-w_{i}\left(x_{0}\right)}{d}=0, i<n ; \quad \text { and } w_{n n}\left(y_{0}\right)=\varphi^{\prime} ;
$$

and

$$
-w_{i n}\left(x_{0}\right)-\frac{w_{i}\left(y_{0}\right)-w_{i}\left(x_{0}\right)}{d}=0, i<n ; \quad \text { and } w_{n n}\left(x_{0}\right)=\varphi^{\prime} .
$$

The second derivative computed in the direction $\left(e_{n},-e_{n}\right)$ is as follows (noting that $\frac{y-x}{|y-x|}$ does not change):

$$
w_{n n n}\left(y_{0}\right)-w_{n n n}\left(x_{0}\right) \leq 2 \varphi^{\prime \prime} .
$$

\footnotetext{
${ }^{4}$ This was until recently the best-known estimate for the general case of convex domains, and was proved by $\mathrm{Yu}$ and Zhong [77] using gradient estimate techniques, essentially as in [80].
} 
The second derivative inequality in the direction $\left(e_{i}, e_{i}\right)$ is as follows (in this case both $\frac{y-x}{|y-x|}$ and $|y-x|$ do not change):

$$
w_{i i n}\left(y_{0}\right)-w_{i i n}\left(x_{0}\right) \leq 0 .
$$

Now we compute the time derivative: Since $w=\log u$ we have

$$
\frac{\partial w}{\partial t}=\Delta w+|\nabla w|^{2}-V .
$$

Differentiating this, we obtain the following equation for derivatives of $w$ :

$$
\frac{\partial}{\partial t} D_{i} w=\Delta D_{i} w+2 D_{k} w D_{k} D_{i} w-D_{i} V .
$$

This gives the following time derivative inequality:

$$
\begin{aligned}
0 \leq & w_{n n n}\left(y_{0}\right)-w_{n n n}\left(x_{0}\right)+\sum_{i<n}\left(w_{i i n}\left(y_{0}\right)-w_{i i n}\left(x_{0}\right)\right)+2\left(\left.w_{n} w_{n n}\right|_{y_{0}}-\left.w_{n} w_{n n}\right|_{x_{0}}\right) \\
& +2 \sum_{i<n}\left(\left.w_{i} w_{i n}\right|_{y_{0}}-\left.w_{i} w_{i n}\right|_{x_{0}}\right)-\left(D_{n} V\left(y_{0}\right)-D_{n} V\left(x_{0}\right)\right)-2 \frac{\partial \varphi}{\partial t} \\
\leq & 2 \varphi^{\prime \prime}+2 \varphi^{\prime}\left(w_{n}\left(y_{0}\right)-w_{n}\left(x_{0}\right)\right)-\frac{2}{d} \sum_{i<n}\left(w_{i}\left(y_{0}\right)-w_{i}\left(x_{0}\right)\right)^{2}-2 \frac{\partial \varphi}{\partial t}
\end{aligned}
$$

$$
\leq 2 \varphi^{\prime \prime}+4 \varphi \varphi^{\prime}-2 \frac{\partial \varphi}{\partial t}
$$

where in obtaining the second inequality we used the inequality (61) in the first two terms, (62) in the second term, the $n$ components of identities (59) and (60) in the third term, and the $i$ components in the fourth term, and we observed that $D_{n} V\left(y_{0}\right)-D_{n} V\left(x_{0}\right) \geq 0$ since $V$ is convex ${ }^{5}$. In obtaining the last line we observed that $w_{n}\left(y_{0}\right)-w_{n}\left(x_{0}\right)=2 \varphi$ since equality holds in (58) at $\left(x_{0}, y_{0}\right)$. This shows that the modulus of concavity $\sigma$ is a viscosity subsolution of the one-dimensional equation

$$
\sigma_{t}=\sigma^{\prime \prime}+2 \sigma \sigma^{\prime}
$$

Observing that in the one-dimensional case we have equality in every inequality we have used, we have an interpretation of equation (66): This is the equation satisfied by the derivative of the logarithm of a positive solution of the one-dimensional heat equation. In particular, we can find a positive even solution $f$ of the one-dimensional Dirichlet heat equation on $[-D / 2, D / 2]$ such that $f^{\prime}(s, 0) / f(s, 0) \geq \sigma(s, 0)$ for all $s \in(-D / 2, D / 2)$. Then the comparison principle implies that $\sigma(s, t) \leq f^{\prime}(s, t) / f(s, t)$ for all $(s, t) \in(0, D / 2) \times[0, \infty)$. In particular, we know that $f(., t)$ as asymptotic to $C \mathrm{e}^{-\pi^{2} / D^{2}} \cos (\pi s / D)$ as $t \rightarrow \infty$, so we have

$$
\lim _{t \rightarrow \infty} \sigma(s, t) \leq-\frac{\pi}{D} \tan \left(\frac{\pi s}{D}\right) .
$$

\footnotetext{
${ }^{5}$ In case the potential is not convex, but there is an inequality on this difference (i.e. a modulus of convexity for $V$ ), then a sharp estimate can also be obtained [8].
} 
In particular, in the case where $u(x, t)=u_{0}(x) \mathrm{e}^{-\lambda_{0} t}$, we have $\sigma(s, t)=\sigma(s, 0)$ for all $t$ and hence

$$
\left(\nabla \log u_{0}(y)-\nabla \log u_{0}(x)\right) \cdot \frac{y-x}{|y-x|} \leq-\frac{\pi}{D} \tan \left(\frac{\pi s}{D}\right),
$$

which is a sharp estimate on the log-concavity of the first Dirichlet eigenfunction on a convex domain with convex potential (sharp in the sense that equality holds in the one-dimensional setting).

Substituting the estimate (68) into the inequality (56), we obtain a sharp result on the modulus of continuity $\omega$ of $v$ : Precisely, $\omega$ satisfies

$$
\omega_{t}(s, t) \leq \omega^{\prime \prime}(s, t)-\frac{2 \pi}{D} \tan \left(\frac{\pi s}{D}\right) \omega^{\prime}
$$

in the viscosity sense. Thus we can compare $\omega$ with an exact solution $C \mathrm{e}^{-\frac{3 \pi^{2}}{D^{2}} t} \sin \left(\frac{\pi s}{D}\right)$ of $(69)$ and deduce a sharp exponential decay rate on $\omega$, implying a sharp lower bound on the difference $\lambda_{1}-\lambda_{0}$ :

$$
\lambda_{1}-\lambda_{0} \geq \frac{3 \pi^{2}}{D^{2}} .
$$

The interested reader should also see the papers [68] and [76] where the ideas presented here are clarified and extended. The fundamental gap problem seems to be one which is naturally amenable to probabilistic arguments, and such an approach (which uses the so-called 'coupling method' developed by $\mathrm{Mu}-\mathrm{Fa}$ Chen and Fengyu Wang, but still relies on some of the key estimates above) was provided in [47]. The same methods were applied to prove a sharp gap result for Schrödinger and diffusion operators on abstract Wiener spaces in [48].

There are still a number of interesting and difficult problems remaining around the fundamental gap: For example, what is the influence of a convex potential when the boundary condition is Neumann or Robin? Is there a sharp gap estimate for convex domains in the sphere or in hyperbolic space, and more generally can one expect a useful lower bound on the gap on a strongly convex Riemannian manifold by controlling curvature, or is it necessary to control higher derivatives of curvature as well?

\section{Isoperimetric profiles under Ricci flow}

Now I will describe a series of results relating to geometric evolution equations, which have a similar approach to some of the results I have described above for scalar heat equations. This work was carried out together with Paul Bryan [2-4,34]. Our starting point for this work was a series of papers, two by Hamilton [51,52] and one by Huisken [54], for twodimensional Ricci flow and for curve-shortening flow.

I will begin with the situation for Ricci flow: Hamilton considered the (normalised) Ricci flow on the two-dimensional sphere, and proved that the isoperimetric profile remains bounded below by a multiple of the isoperimetric profile of the standard metric if it is initially so. We 
adapted his arguments to prove something more: That the isoperimetric profile is a viscosity subsolution of a particular heat equation, with equality characterising a 'one-dimensional' situation where the surface is a surface of rotation.

Let us begin with the definitions: If $g$ is a metric on $S^{2}$ with area $4 \pi$, we define the isoperimetric profile $\mathcal{I}_{g}:[0,4 \pi] \rightarrow \mathbb{R}$ by

$$
\mathcal{I}_{g}(a)=\inf \left\{|\partial \Omega|: \Omega \subset S^{2},|\Omega|=a\right\},
$$

where we allow $\Omega$ to range over all smoothly bounded subsets of $S^{2}$ for which the area (measured using the metric $g$ ) is equal to $a$. For each $a \in(0,4 \pi)$ there exists an isoperimetric region $\Omega$ which achieves the infimum, and any such region has finitely many components, with non-intersecting boundaries of constant geodesic curvature.

To prove a viscosity-type partial differential inequality for the isoperimetric profile, consider an area $a_{0} \in(0,4 \pi)$ and a time $t_{0}>0$, and suppose $\varphi$ is a function which lies below $\mathcal{I}_{g}$ with equality holding at $\left(a, t_{0}\right)$. That is, we have the inequality

$$
|\partial \Omega|_{g(t)}-\varphi\left(|\Omega|_{g(t)}, t\right) \geq 0
$$

for all smoothly bounded subsets $\Omega$ and all times, with equality holding at $t=t_{0}$ and $\Omega=\Omega_{0}$, where $\Omega_{0}$ is an isoperimetric region with area $a_{0}$ (with respect to $\left.g\left(t_{0}\right)\right)$. Now consider smooth variations $\left\{\Omega_{s}\right\}_{|s|<\delta}$ of the subset $\Omega$, given by moving the boundary in the normal direction with some smooth speed $\eta$. Under any such variation we have a first derivative identity and a second derivative inequality. To compute these, we first find the rate of change of the area and the length of the boundary:

$$
\frac{\partial}{\partial s}\left|\Omega_{s}\right|=\int_{\partial \Omega_{s}} \eta, \quad \text { and } \quad \frac{\partial}{\partial s}\left|\partial \Omega_{s}\right|=\int_{\partial \Omega_{s}} k \eta
$$

where $k$ is the geodesic curvature of $\partial \Omega$. In particular, when $s=0$ we have the first derivative identity

$$
\int_{\partial \Omega_{0}} \eta k-\varphi^{\prime} \int_{\partial \Omega_{0}} \eta=0
$$

Since $\eta$ can be taken to be an arbitrary smooth function along $\partial \Omega_{0}$, we deduce that $\partial \Omega_{0}$ has constant curvature $k=\varphi^{\prime}$. Now we compute second variations, but here we only need to compute a particular variation, corresponding to $\eta=1$ (we have in mind the model case here, consisting of surfaces of rotation and where the isoperimetric regions are bounded by circles of rotation, so that moving with constant $\eta$ takes us through a family of isoperimetric regions and we have equality in the second derivative inequality). In this case we have $\partial_{s}\left|\Omega_{s}\right|=\left|\partial \Omega_{s}\right|$, so that $\partial_{s}^{2}\left|\Omega_{s}\right|=\int_{\partial \Omega_{s}} k=$ $\varphi^{\prime} \varphi$. To compute the second variation of the length of the boundary, we write $\partial_{s}\left|\partial \Omega_{s}\right|=\int_{\partial \Omega_{s}} k$, and apply the Gauss-Bonnet theorem to rewrite 
this as $\chi(\Omega)-\int_{\Omega_{s}} K$, where $K$ is the Gauss curvature of the metric $g\left(t_{0}\right)$. Differentiating this gives

$$
\left.\partial_{s}^{2}\left|\partial \Omega_{s}\right|\right|_{s=0}=-\int_{\partial \Omega_{0}} K
$$

Now we combine these identities to give the second variation inequality:

$$
-\int_{\partial \Omega_{0}} K-\varphi\left(\varphi^{\prime}\right)^{2}-\varphi^{\prime \prime} \varphi^{2} \geq 0
$$

Now we turn to the time derivative: Under the normalised (area-preserving) Ricci flow for a metric of area $4 \pi$ on $S^{2}$, the metric evolves by the equation

$$
\frac{\partial g}{\partial t}=-2(K-1) g \text {. }
$$

Consequently we have the following evolution equations for the area $|\Omega|_{g(t)}$ and the boundary length $|\partial \Omega|_{g(t)}$ :

$$
\frac{\partial}{\partial t}\left|\Omega_{0}\right|_{g(t)}=\frac{\partial}{\partial t} \int_{\Omega_{0}} \sqrt{\operatorname{det} g} d x^{1} d x^{2}=-2 \int_{\Omega_{0}}(K-1)=-2 \int_{\Omega_{0}} K+2 a ;
$$

and

$$
\frac{\partial}{\partial t}\left|\partial \Omega_{0}\right|_{g(t)}=\frac{\partial}{\partial t} \int_{\partial \Omega_{0}} \sqrt{g(T, T)}=-\int_{\partial \Omega_{0}}(K-1)=-\int_{\partial \Omega_{0}} K+\varphi .
$$

Everything here can be expressed as an integral over the boundary curve, since we can apply the Gauss-Bonnet theorem to write $\int_{\Omega_{0}} K=2 \pi \chi\left(\Omega_{0}\right)-$ $\int_{\partial \Omega_{0}} k=2 \pi \chi\left(\Omega_{0}\right)-\varphi \varphi^{\prime}$. The only difficulty here is the appearance of the Euler characteristic of $\Omega_{0}$ : In order to compare with the one-dimensional situation we need to ensure that the Euler characteristics are the same, and we can do this as follows: Assume that $\varphi$ is defined on all of $[0,4 \pi]$, is non-negative and strictly concave ${ }^{6}$. Then we claim that $\Omega_{0}$ can only have one connected component: For if there were more than one, we could write $\Omega_{0}=A \cup B$ with $A$ and $B$ disjoint, and then we would have

$$
\begin{aligned}
\varphi(|A|+|B|) & =\varphi\left(\left|\Omega_{0}\right|\right) \\
& =\left|\partial \Omega_{0}\right| \\
& =|\partial A|+|\partial B| \\
& \geq \varphi(|A|)+\varphi(|B|) \\
& >\varphi(0)+\varphi(|A|+|B|) \\
& \geq \varphi(|A|+|B|),
\end{aligned}
$$

\footnotetext{
${ }^{6}$ In making this assumption, we must be satisfied with a weaker conclusion: Rather than proving that the isoperimetric profile is a viscosity supersolution of the resulting equation at every point, we only prove it at exposed points of the convex envelope of the isoperimetric profile. This is enough to carry out comparisons with particular smooth convex subsolutions of the equation, such as those we construct from the sausage model below.
} 
a contradiction. This shows that both $\Omega_{0}$ and $S^{2} \backslash \Omega_{0}$ are connected (since the latter is also an isoperimetric region), and it follows that both are simply connected and have $\chi=1$. This gives the following time inequality:

$$
-\int_{\partial \Omega_{0}} K+\varphi-\varphi^{\prime}\left(-4 \pi+2 \varphi \varphi^{\prime}+2 a\right)-\varphi_{t} \leq 0 .
$$

Combining this with the second variation inequality (76), we obtain the following:

$$
\begin{aligned}
\varphi_{t} & \geq-\int_{\partial \Omega_{0}} K+\varphi-2 \varphi^{\prime} a+4 \pi \varphi^{\prime}-2 \varphi\left(\varphi^{\prime}\right)^{2} \\
& \geq \varphi^{2} \varphi^{\prime \prime}-\varphi\left(\varphi^{\prime}\right)^{2}+\varphi+\varphi^{\prime}(4 \pi-2 a) .
\end{aligned}
$$

That is, the isoperimetric profile is a viscosity solution of the above inequality at the exposed points of its convex envelope. One can now recover Hamilton's estimate by doing a quick computation to check that any positive multiple (less than 1) of the isoperimetric profile of the standard metric is a subsolution of the above equation. However, we can deduce a much nicer result:

Equality holds in the above computation in the case of surfaces of rotation evolving by Ricci flow (provided the isoperimetric profile is concave). In fact, given an arbitrary rotationally symmetric solution of the Ricci flow on $S^{2}$, we can construct a smooth solution to the above equation (with equality rather than inequality) by taking the family of discs bounded by the circles of rotation, and taking $\varphi$ to be the function which gives the length of the boundary circle as a function of the area. The assumption that the Gauss curvature of the rotational solution is positive guarantees that the function $\varphi$ is strictly concave.

We conclude: If $\bar{g}$ is a metric with axial symmetry and positive Gauss curvature on $S^{2}$ of area $4 \pi$, evolving by normalised Ricci flow, and $g$ is an arbitrary solution of normalised Ricci flow on $S^{2}$ with area $4 \pi$, and $\mathcal{I}_{g(0)} \geq \mathcal{I}_{\bar{g}(0)}$, then $\mathcal{I}_{g(t)} \geq \mathcal{I}_{\bar{g}(t)}$ for all positive $t$ (as long as both solutions exist).

There is a beautiful exact solution of the Ricci flow on $S^{2}$, known variously as the Rosenau solution [71], the King solution [58], the FateevOnofri-Zamolodchikov solution [45], and the sausage model. This exists for all time $t$ under the normalised Ricci flow, approaches the standard metric as $t \rightarrow \infty$, and approaches a long thin sausage as $t \rightarrow-\infty$. In particular, for an arbitrary smooth metric $g(0)$ on $S^{2}$, the isoperimetric profile of $g(0)$ is bounded below by that of the King solution for sufficiently negative $t$, and we obtain a useful comparison for positive times.

A remarkable consequence of this (which cannot be obtained by Hamilton's earlier comparison with a multiple of the isoperimetric profile of the sphere) is that we also obtain very strong control on the curvature: The idea is that large Gauss curvature at some point means that lengths of small circles are relatively short, and this implies an asymptotic expansion for the 
isoperimetric profile near $a=0$ : The isoperimetric profile of the flat metric on $\mathbb{R}^{2}$ is $\sqrt{4 \pi a}$, and in general we have $\mathcal{I}_{g}(a)=\sqrt{4 \pi a}\left(1-\frac{K_{\max }}{8 \pi} a+o(a)\right)$ as $a \rightarrow 0$, where $K_{\max }$ is the maximum of the Gauss curvature of $g$. It follows that if $\mathcal{I}_{g} \geq \mathcal{I}_{\bar{g}}$, then $K_{\max }(g) \leq K_{\max }(\bar{g})$. Since we know the King solution exactly, and the curvature decays exponentially to 1 , we deduce that for an arbitrary smooth solution of Ricci flow on $S^{2}$ we have $K \leq 1+C \mathrm{e}^{-2 t}$ for some $C$. Note that this gives only an upper bound, but under Ricci flow we always have a good lower bound of the form $K \geq-\frac{1}{2 t}$, and by Gauss-Bonnet we know that $\frac{1}{4 \pi} \int K=1$, so the upper bound decaying to 1 has strong consequences. A very easy proof of convergence to the constant curvature metric results.

In recent work [34], Bryan has extended the method to handle the case of Ricci flow on arbitrary compact surfaces. In this case the argument to show that the isoperimetric regions are simply connected breaks down, but one can instead work with the isoperimetric profile of the universal cover. Again the isoperimetric profile is a viscosity supersolution of a certain equation, and by constructing suitable barriers for this equation one can deduce good control both on the isoperimetric profile and the maximum of curvature. Some care must be taken to ensure the comparison holds, by establishing some asymptotics for the isoperimetric profile at infinity.

The method outlined above of establishing that the isoperimetric profile satisfies a differential inequality in a viscosity sense has been adapted by Lei $\mathrm{Ni}$ and Kui Wang [69] to the situation of (static) metrics satisfying lower Ricci curvature bounds in any dimension. In particular the method allows an elegant way to establish the isoperimetric profile bounds of Levy-Gromov [49] and Bérard-Besson-Gallot [26], as well as some new comparison results.

\section{Isoperimetric profile under curve shortening flow}

There is a similar story for the evolution of curves in the plane under curve-shortening flow, although the details end up being somewhat more complicated: Such a curve is the boundary of a bounded open region $\Omega$, and one can consider the isoperimetric profile of this region as the curve evolves: We normalise the regions to have area $\pi$, and then the isoperimetric profile is defined as follows:

$$
\mathcal{I}_{\Omega}(a):=\inf \left\{L\left[\partial_{\Omega} A\right]: A \subset \Omega, A=a\right\} .
$$

Here $A$ can be assumed to be subset of $\Omega$ with smooth boundary curve which meets the evolving curve $\partial \Omega$ orthogonally. As before, for any $a \in(0, \pi)$ there exists an isoperimetric region $A$ attaining the infimum, and this has finitely many components, with disjoint boundary curves meeting $\partial \Omega$ orthogonally and having constant geodesic curvature.

Hamilton also considered this isoperimetric profile [52], and proved that a lower bound by a multiple of the isoperimetric profile of the round ball is maintained under the flow. Paul Bryan and I showed that the isoperimetric profile is in fact a viscosity supersolution of a certain one-dimensional heat 
equation, and that equality holds for the isoperimetric profiles of certain model curves: Curves which are symmetric in both coordinate axes, and have no critical points of curvature other than the four which occur at the intersections with the coordinate axes.

Here is a sketch of the argument: If $\varphi$ is a smooth function which lies below the isoperimetric profile, with equality at some area $a_{0} \in(0, \pi)$ at some positive time $t_{0}$, we let $A_{0}$ be an isoperimetric domain in $\Omega_{t_{0}}$, so that we have the inequality

$$
L\left[\partial_{\Omega_{t}} A\right] \geq \varphi(|A|, t)
$$

for any (smoothly bounded) subset $A$ of $\Omega_{t}$, for any $t \leq t_{0}$, and we have equality when $A=A_{0}$ and $t=t_{0}$. As before we make the assumption that $\varphi$ is concave, in which case the isoperimetric region $A_{0}$ is necessarily connected and has connected complement, from which it follows that it has a single boundary curve which is a segment of a circle meeting $\partial \Omega_{0}$ orthogonally. Now consider any smooth family of regions $\left\{A_{s}\right\}$ for small $s$, agreeing with $A_{0}$ when $s=0$ : The inequality (83) implies the identities $\left.\frac{\partial}{\partial s}\left(L\left[\partial A_{s}\right]-\varphi\left(\left|A_{s}\right|, t_{0}\right)\right)\right|_{s=0}=0$ and $\left.\left(\frac{\partial}{\partial s}\right)^{2}\left(L\left[\partial A_{s}\right]-\varphi\left(\left|A_{s}\right|, t_{0}\right)\right)\right|_{s=0} \leq 0$. The first of these can be written as follows:

$$
\int_{\partial A} k \eta-\varphi^{\prime} \int_{\partial A} \eta=0
$$

where $\eta$ is the normal component of motion of the boundary curve $\partial A$. Since $\eta$ can be taken to be an arbitrary smooth function, we deduce that $\partial A$ has curvature $k$ equal to $\varphi^{\prime}$. This means that the curve $\partial A$ is completely determined, up to congruence, by the function $\varphi$ and the value $a_{0}$, since the length and the curvature are both fixed. It follows that there is a unique choice of $\eta$ which corresponds to moving the endpoints of $\partial A$ at unit speed outwards, such that the curvature remains constant and the ends remain orthogonal (to first order), and this is determined entirely by $a_{0}, \varphi\left(a_{0}, t_{0}\right)$, and $\varphi^{\prime}\left(a_{0}, t_{0}\right)$. Under this variation one can compute the second variation inequality: The second variation of the length includes a contribution coming from the curvatures of $\partial \Omega_{t_{0}}$ at the endpoints with the remaining terms determined entirely by the boundary curve itself, which is in turn determined by $a_{0}, \varphi$ and $\varphi^{\prime}$; the second variation of the area is also completely determined by these. Thus the second variation inequality has the following form:

$$
-k\left(p_{+}\right)-k\left(p_{-}\right)+\mathcal{F}\left(a_{0}, \varphi, \varphi^{\prime}, \varphi^{\prime \prime}\right) \geq 0,
$$

where $p_{+}$and $p_{-}$are the endpoints of $\partial A$, and $\mathcal{F}$ is a smooth function that we do not need to determine, except to observe that in the special case of a curve with symmetries in both axes and exactly four vertices, equality holds in the inequality. Similarly, we have a time derivative inequality: Under the normalised curve shortening flow $\frac{\partial X}{\partial t}=-(k-1) \nu$ (which keeps the enclosed area equal to $2 \pi)$, the rate of change of the boundary length $L[\partial A]$ is equal to $-k\left(p_{+}\right)-k\left(p_{-}\right)+2$, while the rate of change of the enclosed area is equal 
to twice $a_{0}$ minus the integral of curvature around $\partial \Omega_{t_{0}}$ from $p_{-}$to $p_{+}$. By the theorem of turning tangents, the latter is equal to $2 \pi$ minus $\pi$ (for the two right angles at the endpoints) minus the integral of curvature $k=\varphi^{\prime}$ along $\partial A$, which is just $\varphi \varphi^{\prime}$. Thus again, everything can be expressed in terms of $a_{0}, \varphi$ and $\varphi^{\prime}$, and we have an inequality of the form

$$
-k\left(p_{+}\right)-k\left(p_{-}\right)-\varphi_{t}+\mathcal{G}\left(a_{0}, \varphi, \varphi^{\prime}\right) \leq 0,
$$

where $\mathcal{G}$ is again a smooth function of its arguments, and equality holds in the symmetric case. Combining (85) and (86) we can cancel the curvature terms, and produce the inequality

$$
\varphi_{t} \geq \tilde{\mathcal{F}}\left(\varphi^{\prime \prime}, \varphi^{\prime}, \varphi, a_{0}\right),
$$

for some function $\tilde{\mathcal{F}}$. That is to say, the isoperimetric profile is a viscosity supersolution of a certain (fully nonlinear) parabolic differential equation. The important thing for us is that in the symmetric case, all of the inequalities hold with equality, so that the isoperimetric profile of a symmetric solution of normalised curve shortening flow with four vertices satisfies (87) with equality. In particular, if the isoperimetric profile of the initial curve lies above that of a symmetric curve, and we evolve both by the normalised curve shortening flow, then the inequality is maintained.

Just as in the Ricci flow case, there is a beautiful exact solution of the curve-shortening flow which is symmetric in both axes and has four vertices. This is known as the paperclip solution, or the Angenent oval. For the normalised flow it exists for all time and converges exponentially to the unit circle, and as $t$ becomes very negative it becomes very long and thin, with the isoperimetric profile converging to zero. It follows that for any smooth initial embedded curve, we can bound the isoperimetric profile of the enclosed region from below by that of a paperclip for sufficiently negative $t$, and the above comparison result implies that the inequality is maintained for positive times. In particular, the isoperimetric profile converges to that of the unit ball, and it follows also that the curvature has an upper bound which converges exponentially to 1 . In this case we can also get a lower bound on the curvature, by carrying out a similar (slightly modified) argument to control the isoperimetric profile of the exterior region. This produces a very direct proof of Grayson's theorem, that the curve shortening flow evolves an arbitrary embedded closed curve in the plane to a circle, modulo scaling.

\section{Chord-arc profile under curve shortening flow}

Paul Bryan and I also looked at a different 'isoperimetric profile' to control the behaviour of curve-shortening flow: The chord-arc profile. Again, our estimate was inspired by earlier work, this time by Huisken [54]. The chord-arc profile of an embedded closed curve $\gamma$ in the plane is defined by

$$
\mathcal{C}(\ell)=\inf \{|p-q|: p, q \in \gamma, \ell(p, q)=\ell\},
$$


where $\ell(p, q)$ is the distance from $p$ to $q$ along the curve $\gamma$. Huisken proved the following: If the evolving curves are rescaled to have fixed length equal to $2 \pi$, and the chord-arc profile of the initial curve is bounded below by a multiple of that of the round circle, then this remains true at later times under the curve-shortening flow. Our result strengthens this, proving that the chord-arc profile is a viscosity supersolution of a certain one-dimensional parabolic equation. In this case, however, our result is somewhat less sharp, since we apply the inequality $\ell \int k^{2} \geq\left(\int k\right)^{2}$ over segments of the curve, and this is an inequality which does not hold in any situation other than the circle, in contrast to the situation with the isoperimetric profile where all the inequalities we applied held with equality in the case of symmetric curves. Nevertheless, we were able to construct explicit barriers for the resulting equation, which were sufficient to imply that the chord-arc profile converges (under a suitably normalised curve shortening flow) to that of the unit circle, and it follows that we have a bound of the form $k^{2} \leq 1+C \mathrm{e}^{-2 t}$ for some $C$. The problem of strengthening the argument to make it a true comparison result, analogous to that which holds for the isoperimetric profile, is an interesting one and involves some interesting conjectured inequalities in curve geometry. One reason to try to do this is that the argument would also apply for flows of embedded curves by powers of curvature, and one could hope to prove versions of Grayson's theorem for such flows.

$\mathrm{Su}$ Tao, while still an undergraduate student at Tsinghua University, used these chord-arc profile ideas to provide an elegant proof of Smale's theorem on diffeomorphisms of the disk (and consequently also for diffeomorphisms of the 2-sphere $S^{2}$ ). The idea here is as follows: Given a diffeomorphism of the unit disc which fixes the boundary, we can first compose with a canonical conformal 'boost' so that the diffeomorphism fixes the origin. Then a smooth correction can be made to ensure that the diffeomorphism is the identity near the boundary, and equal to a rotation near the origin. Now conjugate by a conformal map from the punctured plane to the cylinder, to produce a diffeomorphism $\Psi$ from $S^{1} \times \mathbb{R}$ to itself, which fixes all of the circles $S^{1} \times\{s\}$ for $s \geq 1$, and rotates the circles $S^{1} \times\{s\}$ through a fixed angle for $s$ very negative. Now consider the family of circles $\Psi\left(S^{1} \times\{s\}\right)$, and evolve them by curve shortening flow. A modified version of the chord-arc estimates apply to prove that these evolve to the product circles $S^{1} \times\{\mu(s)\}$ as $t \rightarrow \infty$, for some increasing smooth function $\mu$ which is equal to the identity near infinity. One can now produce a simple deformation of the original diffeomorphism to the identity map, as the problem reduces to a simple one of reparametrisations on circles. See [22] for further details on this argument.

It would be very interesting to find other situations where these kinds of arguments on isoperimetric profiles can be made to work: One promising candidate is Ricci flow in three dimensions, where one could still hope to apply the Gauss-Bonnet theorem to the boundaries of isoperimetric regions to obtain control over the isoperimetric profile (some hints of the kinds of arguments involved can be found in [50]). Could one perhaps find a proof 
of Hamilton's theorem on three-manifolds with positive Ricci curvature using isoperimetric arguments? And would this allow us to weaken the Ricci curvature assumption to an assumption on isoperimetric profiles or isoperimetric regions? Also possibly interesting would be applications to Kähler-Ricci flow or to Yamabe flow, particularly in conformally flat cases.

\section{Higher derivative estimates}

In the next few sections I want to describe a non-collapsing estimate for hypersurfaces moving by curvature, and the arguments developed for that purpose which were later used by Brendle to prove the Lawson conjecture for minimal surfaces in the three-sphere. As a prelude to this I will first describe some analogous ideas for scalar equations.

First consider the heat equation, for simplicity with periodic initial data on Euclidean space (so invariant under the group of translations in some lattice $\Gamma$ ). We can define a variation on the modulus of continuity which serves to control higher derivatives of the solution, as follows: Define the $k$ th order modulus of continuity $\omega_{k}(s, t)$ of a solution $u: \mathbb{R}^{n} \times[0, \infty) \rightarrow \mathbb{R}$ of the heat equation to be

$\omega_{k}(s)=\sup \left\{\frac{1}{2}\left|u(y, t)-\sum_{i=0}^{k} \frac{1}{i !} D^{(i)} u\right|_{(x, t)}(y-x, \ldots, y-x)|:| y-x \mid=2 s\right\}$.

That is, $\omega_{k}$ measures the deviation from the $k$ th order Taylor approximation of the functions about $x$, in terms of the distance from $x$. As I will show, these higher moduli of continuity can be controlled in a very similar way to the usual modulus of continuity, and turn out to be viscosity subsolutions of rather simple one-dimensional heat equations.

To see this, suppose $\varphi$ is a function lying above $\omega_{k}$, with equality at $\left(s_{0}, t_{0}\right)$ for some $s_{0}>0$, and let $x_{0}$ and $y_{0}$ be points where the supremum is attained in the definition (89) at $\left(s_{0}, t_{0}\right)$. Assume without loss of generality that $u\left(y_{0}, t_{0}\right)$ lies above the $k$ th Taylor polynomial of $u$ at $\left(x_{0}, t_{0}\right)$, so that we have the inequality

$$
u(y, t)-\left.\sum_{\ell=0}^{k} \frac{1}{\ell !} D^{(\ell)} u\right|_{(x, t)}(y-x, \ldots, y-x)-2 \varphi\left(\frac{|y-x|}{2}, t\right) \leq 0
$$

for all $x, y \in \mathbb{R}^{n}$ and all $t \in\left[0, t_{0}\right]$, with equality at $\left(x_{0}, y_{0}, t_{0}\right)$. As usual we choose a basis with $e_{n}=\frac{y-x}{|y-x|}$. Then moving $y$ in the $e_{i}$ direction gives the following first order identities:

$$
\left.D_{i} u\right|_{\left(y_{0}, t_{0}\right)}-\left.\sum_{\ell=1}^{k} \frac{1}{(\ell-1) !} D^{(\ell)} u\right|_{\left(x_{0}, t_{0}\right)}\left(e_{i}, y-x, \ldots, y-x\right)-\varphi^{\prime} \delta_{i n}=0 .
$$


Differentiating (90) in the $x^{i}$ direction gives the following, since many cancellations occur:

$$
-\left.\frac{1}{k !} D^{(k+1)} u\right|_{\left(x_{0}, t_{0}\right)}\left(e_{i}, y-x, \ldots, y-x\right)+\varphi^{\prime} \delta_{i n}=0 .
$$

We also have second variation inequalities: If we move $x$ and $y$ together in the $e_{i}$ direction with $i<n$, then $y-x$ does not change, and we find

$$
0 \geq\left. u_{i i}\right|_{\left(y_{0}, t_{0}\right)}-\left.\sum_{\ell=0}^{k} \frac{1}{\ell !} D^{(\ell)} u_{i i}\right|_{\left(x_{0}, t_{0}\right)}(y-x, \ldots, y-x) .
$$

If we move $x$ and $y$ apart (so that $\dot{x}=-e_{n}$ and $\dot{y}=e_{n}$ ) we observe that $y-x$ changes at rate $2 e_{n}$, and get the inequality

$$
\begin{aligned}
0 \geq & \left.u_{n n}\right|_{\left(y_{0}, t_{0}\right)}-\left.\sum_{\ell=0}^{k} \frac{1}{\ell !} D^{(\ell)} u_{n n}\right|_{\left(x_{0}, t_{0}\right)}(y-x, \ldots, y-x) \\
& +\left.2 \sum_{\ell=1}^{k} \frac{1}{(\ell-1) !} D^{(\ell)} u_{n}\right|_{\left(x_{0}, t_{0}\right)}\left(y-x, \ldots, y-x, 2 e_{n}\right) \\
& -\left.\sum_{\ell=2}^{k} \frac{1}{(\ell-2) !} D^{(\ell)} u\right|_{\left(x_{0}, t_{0}\right)}\left(y-x, \ldots, y-x, 2 e_{n}, 2 e_{n}\right)-2 \varphi^{\prime \prime} \\
= & \left.u_{n n}\right|_{\left(y_{0}, t_{0}\right)}-\left.\sum_{\ell=0}^{k} \frac{1}{\ell !} D^{(\ell)} u_{n n}\right|_{\left(x_{0}, t_{0}\right)}(y-x, \ldots, y-x) \\
& +\frac{4}{\left|y_{0}-x_{0}\right|} \frac{1}{(k-1) !} D^{(k)} u_{n}(y-x, \ldots, y-x)-2 \varphi^{\prime \prime} \\
= & \left.u_{n n}\right|_{\left(y_{0}, t_{0}\right)}-\left.\sum_{\ell=0}^{k} \frac{1}{\ell !} D^{(\ell)} u_{n n}\right|_{\left(x_{0}, t_{0}\right)}(y-x, \ldots, y-x) \\
& +\frac{2 k}{s_{0}} \varphi^{\prime}-2 \varphi^{\prime \prime}
\end{aligned}
$$

where we used the first derivative identity (92) and the fact that $\left|y_{0}-x_{0}\right|=$ $2 s_{0}$ to produce the last equality. Adding the inequalities (93) for $i<n$ and (94), we obtain

$$
0 \geq\left.\Delta u\right|_{\left(y_{0}, t_{0}\right)}-\left.\sum_{\ell=0}^{k} \frac{1}{\ell !} D^{(\ell)} \Delta u\right|_{\left(x_{0}, t_{0}\right)}(y-x, \ldots, y-x)+\frac{2 k}{s_{0}}-2 \varphi^{\prime \prime} .
$$

Finally, we have from the time derivative inequality

$$
\left.\Delta u\right|_{\left(y_{0}, t_{0}\right)}-\left.\sum_{\ell=0}^{k} \frac{1}{\ell !} D^{(\ell)} \Delta u\right|_{\left(x_{0}, t_{0}\right)}(y-x, \ldots, y-x)-2 \varphi_{t} \geq 0,
$$

from which it follows that

$$
\varphi_{t} \leq \varphi^{\prime \prime}-\frac{k}{s_{0}} \varphi^{\prime}
$$


meaning that the $k$ th order modulus of continuity $\omega_{k}$ is a viscosity subsolution of the heat equation $\omega_{t}=\omega^{\prime \prime}-\frac{k}{s} \omega^{\prime}$. We can now produce bounds on $\omega_{k}$ by producing suitable supersolutions of the same equation. In particular we want to find a supersolution which lies above the initial data of $\omega_{k}$, and we also need to ensure that the comparison holds near infinity. If we assume that the derivatives of $u$ up to order $k$ are bounded, this means we need to find a supersolution which starts greater than $C s^{k}$ at $t=0$ and remains greater than $C s^{k}$ for $s$ large for positive $t$, but to deduce a bound on the $(k+1)$-st derivative for positive times we need the supersolution to be like $s^{k+1}$ for $s$ small. One can produce by ODE methods a self-similar solution of the form $f(s, t)=t^{k / 2} F(x / \sqrt{t})$, where $F(\xi)$ is asymptotic to a multiple of $\xi^{k}$ at infinity, and to $\xi^{k+1}$ as $\xi \rightarrow 0$, and using this barrier gives that the order $k+1$ derivative of $u$ is bounded for positive times, with a bound proportional to $1 / \sqrt{t}$.

\section{Equations with bounded coefficients}

The argument as presented in the previous section has some drawbacks, notably that applications to more general equations require a lot of smoothness of the coefficients if $k$ is large. One can formulate arguments which use more than two points which avoid differentiating the coefficients, but this becomes more complicated. I will present here a simple case of an argument which Julie Clutterbuck and I found [10] to prove regularity results which do not depend on regularity of coefficients: Consider a uniformly parabolic linear equation in one space variable, and for ease of presentation consider the simplest possible case without lower order terms:

$$
u_{t}=a(x, t) u^{\prime \prime}
$$

We assume that the coefficient $a(x, t)$ is smooth, but derive estimates which depend only on pointwise upper and lower bounds for the coefficients and not on any higher regularity, so the estimate can be extended to non-smooth coefficient equations in many cases. In this setting it is very easy to prove a good modulus of continuity bound by a simple version of the method described in section 2 , and it is known that in general the best regularity that can be expected is some Hölder continuity of the gradient. For simplicity (to avoid boundary issues) we consider the case of a spatially periodic solution, and define a function which captures the deviation of the solution at one point from the linear function which agrees at two other points: We set

$$
\begin{aligned}
& \sigma(\xi, \eta, t) \\
& \quad:=\sup \left\{\left|u(y, t)-\frac{y-x}{z-x} u(z, t)-\frac{z-y}{z-x} u(x, t)\right|: y-x=\xi, z-y=\eta\right\} .
\end{aligned}
$$

One can characterise the Hölder continuity of the spatial gradient of $u$ in terms of $\sigma: u(., t)$ is in $C^{1, \alpha}$ if and only if $\sigma(\xi, \eta, t)$ is bounded by a 
multiple of $(\xi+\eta)^{1+\alpha}$ for all (small positive) $a$ and $b$. We will show that $\sigma$ satisfies a certain differential inequality (in a viscosity sense) and construct an explicit barrier to bound $\sigma$ and deduce a $C^{1, \alpha}$ bound. Consider a function $\varphi$ which lies above $\sigma$, making contact at $\left(\xi_{0}, \eta_{0}, t_{0}\right)$ for some positive $\xi_{0}$ and $\eta_{0}$ and $t_{0}>0$, and let $x_{0}, y_{0}$ and $z_{0}$ be points where equality is attained. Then we obtain first and second derivative inequalities by differentiating the inequality

$$
\begin{aligned}
Z(x, y, z, t)= & (z-x) u(y, t)-(z-y) u(x, t)-(y-x) u(z, t) \\
& -(z-x) \varphi(y-x, z-y, t) \leq 0,
\end{aligned}
$$

for which equality holds at $x_{0}, y_{0}, z_{0}, t_{0}$. The first derivative identities are as follows (at the point $\left(x_{0}, y_{0}, z_{0}\right)$ :

$$
\begin{aligned}
& Z_{x}=-(z-y) u^{\prime}(x)+u(z)-u(y)+\varphi+(\xi+\eta) \varphi_{\xi}=0 \\
& Z_{y}=(z-x) u^{\prime}(y)-u(z)+u(x)-(\xi+\eta)\left(\varphi_{\xi}-\varphi_{\eta}\right)=0 \\
& Z_{z}=-(y-x) u^{\prime}(z)+u(y)-u(x)-\varphi-(\xi+\eta) \varphi_{\eta}=0
\end{aligned}
$$

where $\xi=y-x$ and $\eta=z-y$. Further differentiation gives the following (for a crude estimate we do not need the full matrix of second derivatives):

$$
\begin{aligned}
& Z_{x x}=-(z-y) u^{\prime \prime}(x, t)-2 \varphi_{\xi}-(\xi+\eta) \varphi_{\xi \xi} \leq 0 ; \\
& Z_{y y}=(z-x) u^{\prime \prime}(y, t)-(\xi+\eta)\left(\varphi_{\xi \xi}-2 \varphi_{\xi \eta}+\varphi_{\eta \eta}\right) \leq 0 ; \\
& Z_{z z}=-(y-x) u^{\prime \prime}(z, t)-2 \varphi_{\eta}-(\xi+\eta) \varphi_{\eta \eta} \leq 0 .
\end{aligned}
$$

We also have the time derivative inequality:

$$
\begin{aligned}
Z_{t}= & (z-x) a(y, t) u^{\prime \prime}(y, t)-(z-y) a(x, t) u^{\prime \prime}(x, t) \\
& -(y-x) a(z, t) u^{\prime \prime}(z, t)-(z-x) \varphi_{t} \geq 0 .
\end{aligned}
$$

Combining these, we obtain the inequality

$\varphi_{t} \leq a(y, t)\left(\varphi_{\xi \xi}-2 \varphi_{\xi \eta}+\varphi_{\eta \eta}\right)+a(x, t)\left(\varphi_{\xi \xi}+\frac{2}{\xi+\eta} \varphi_{\xi}\right)+a(z, t)\left(\varphi_{\eta \eta}+\frac{2}{\xi+\eta} \varphi_{\eta}\right)$.

We assume that the coefficients lie in the range $\lambda \leq a(x, t) \leq \Lambda$ for some positive constants $\lambda$ and $\Lambda$. Since we do not know the specific values the coefficients take in this range, the most we can say for $\varphi$ (as a function of $\xi$ and $\eta$ ) is that it satisfies the following differential inequality:

$$
\begin{gathered}
\varphi_{t} \leq \sup \left\{a_{y}\left(\varphi_{\xi \xi}-2 \varphi_{\xi \eta}+\varphi_{\eta \eta}\right)+a_{x}\left(\varphi_{\xi \xi}+\frac{2 \varphi_{\xi}}{\xi+\eta}\right)+a_{z}\left(\varphi_{\eta \eta}+\frac{2 \varphi_{\eta}}{\xi+\eta}\right):\right. \\
\left.a_{x}, a_{y}, a_{z} \in[\lambda, \Lambda]\right\} .
\end{gathered}
$$

This differential inequality is satisfied (in the viscosity sense) by the function $\sigma$ defined by (99) for any solution of an equation of the form (98) with coefficients in the range $[\lambda, \Lambda]$. To proceed, we try to find a supersolution $f$ of the same equation. For simplicity let us look for a stationary supersolution. Here $f$ should be zero for $\xi=0$ or $\eta=0$, so we can try the simple ansatz 
$f(\xi, \eta)=(\xi+\eta)^{\alpha-1} \xi \eta$. A direct calculation shows that the supremum on the right-hand side of (105) is non-positive for all $\xi, \eta>0$ if we choose $\alpha=\frac{\lambda}{\Lambda}$ (or, working a little harder, the slightly larger exponent $\left.\alpha=\frac{\frac{8 \lambda}{\Lambda}}{3+\sqrt{9+\frac{16 \lambda}{\Lambda}}}\right)$. This shows that Hölder continuity of the gradient with this exponent is preserved under the equation (98), and this can be improved to give a bound on the $C^{1, \alpha}$ norm of a solution for positive times, depending only on an initial $C^{0}$ bound and elapsed time. In [10] we give a more detailed discussion, and address the question of the optimal Hölder exponent for given $\lambda$ and $\Lambda$.

It would be very interesting to use these methods to prove Hölder continuity of solutions of parabolic equations with bounded measureable coefficients in higher dimensions - this is of course a famous result of Krylov and Safonov [62] and an argument by the methods outlined above would very interesting, particularly if it sheds some light on the optimal Hölder exponent ${ }^{7}$.

\section{Upper and lower paraboloids}

Next I will discuss a different construction, which also relates to higher regularity for solutions of heat equations, but this time instead of estimating a modulus of continuity or some generalisation which does not depend on the point on the domain, here we define a function of the point which captures some global information about the solution at nearby points. As we shall see, this idea relates very closely to that which appears in the non-collapsing argument for mean curvature flow, and to that used in Brendle's proof of the Lawson conjecture.

Consider a solution $u: \mathbb{R}^{n} / \Gamma \rightarrow \mathbb{R}$ of a heat equation, and define the following function which gives the Hessian of the smallest paraboloid which lies above the graph of $u$ and touches at a given point:

$$
\begin{aligned}
\bar{h}(x, t) & =\inf \left\{r: u(y, t) \leq u(x, t)+D u(x, t)(y-x)+\frac{r}{2}|y-x|^{2} \text { for all } y \in \mathbb{R}^{n}\right\} \\
& =\sup \left\{2 \frac{u(y, t)-u(x, t)-D u(x, t)(y-x)}{|y-x|^{2}}: y \in \mathbb{R}^{n} \backslash\{x\}\right\} .
\end{aligned}
$$

\footnotetext{
${ }^{7}$ One of many things I learnt from coffee-table discussions at Stanford with David Gilbarg and Leon Simon concerned the problem of estimating the optimal Hölder exponent for the gradient in elliptic equations in two variables, which goes through the route of Beltrami systems where the optimal exponent is known [46, Theorem 12.3]. In this case the best known exponent is $\sqrt{\frac{\lambda}{\Lambda}}$ (see [46, Section 12.2], but - as Leon pointed out to me - the stronger result $\alpha=\sqrt{\frac{\lambda}{\Lambda}}$ instead of $\frac{\lambda}{\Lambda}$ is obtained by using the normalisation $\lambda \Lambda=1$ instead of $\lambda=1$ ). As far as I can tell this is not the optimal exponent for the elliptic case, even though the result for Beltrami systems is sharp, since the examples corresponding to this exponent in the Beltrami case do not correspond to the solution of an elliptic equation. The question of optimal Hölder exponents for linear parabolic equations with bounded measureable coefficients seems much further from resolution by existing techniques.
} 
We will show that $\bar{h}$ satisfies a useful differential inequality (in a viscosity sense as usual, since we cannot expect $\bar{h}$ to be a smooth function). Suppose that $\varphi$ is a smooth function which lies above $\bar{h}$, and makes contact at some point $\left(x_{0}, t_{0}\right)$. We have two possibilities: Either the supremum in the second line of (106) is attained for some $y_{0} \neq x_{0}$, or it is not. In the latter case, the supremum must be attained in a limit as $y \rightarrow x_{0}$, and we must have $\bar{h}\left(x_{0}\right)$ equal to the largest eigenvalue of the Hessian of $D^{2} u\left(x_{0}, t_{0}\right)$. Let $e$ be the corresponding eigenvector. Then we have the inequality

$$
\varphi(x, t) \geq\left. D^{2} u\right|_{(x, t)}(e, e)
$$

for all $(x, t)$, with equality at $\left(x_{0}, t_{0}\right)$. Differentiating this gives second variation and time inequalities

$$
D^{2} \varphi \geq D^{2}\left(D^{2} u(e, e)\right) \quad \text { and } \varphi_{t} \leq \Delta\left(D^{2} u(e, e)\right)
$$

from which we deduce the inequality $\varphi_{t} \leq \Delta \varphi$. Now we deal with the second case, where equality is attained for some $y_{0} \neq x_{0}$ : Then we have the inequality

$$
\varphi(x, t) \geq 2 \frac{u(y, t)-u(x, t)-D u(x, t)(y-x)}{|y-x|^{2}}
$$

for all $y \neq x$ and $t>0$, and equality holds at $\left(x_{0}, y_{0}, t_{0}\right)$. Differentiating by moving both $x$ and $y$ in the same direction $e_{i}$, and observing that $y-x$ does not change and that $\varphi$ depends only on $x$, we obtain the following second derivative inequality:

$$
\frac{\partial^{2} \varphi}{\partial x^{i} \partial x^{j}} \geq 2 \frac{\left.u_{i i}\right|_{\left(y_{0}, t_{0}\right)}-\left.u_{i i}\right|_{\left(x_{0}, t_{0}\right)}-\left.D u_{i i}\right|_{\left(x_{0}, t_{0}\right)}\left(y_{0}-x_{0}\right)}{\left|y_{0}-x_{0}\right|},
$$

and we have the time inequality

$$
\varphi_{t} \leq 2 \frac{\left.\Delta u\right|_{\left(y_{0}, t_{0}\right)}-\left.\Delta u\right|_{\left(x_{0}, t_{0}\right)}-\left.D \Delta u\right|_{\left(y_{0}, t_{0}\right)}\left(y_{0}-x_{0}\right)}{\left|y_{0}-x_{0}\right|^{2}} .
$$

Taking the trace of (110) then gives the inequality

$$
\varphi_{t} \leq \Delta \varphi
$$

proving that the function $\bar{h}$ is a viscosity subsolution of the heat equation. The same argument shows that the largest paraboloid which touches from below defines a viscosity supersolution of the heat equation. The same argument can be made to work for a range of nonlinear equations, with some small modifications.

\section{Inscribed ball curvature and noncollapsing}

The non-collapsing estimate for mean curvature flow in [1] is somewhat analogous to that for the heat equation in the previous section. In this case we consider a compact embedded hypersurface in Euclidean space evolving by mean curvature flow, and consider a function which characterises the curvature of the largest ball in the enclosed region which touches at a 
given point. As observed in [16], this function satisfies a natural differential inequality on the evolving hypersurface, in the viscosity sense.

We can find an expression for this quantity, analogous to (106), as follows: Let $M$ be an embedded hypersurface, and $\Omega$ the enclosed region. We choose the unit normal $\nu$ to point out of $\Omega$. If $B$ is a ball of radius $r$ contained in $\Omega$ and touching at a point $x \in M$, then the centre of $B$ must be at the point $x-r \nu(x)$, and the statement that $B$ is contained in $\Omega$ is equivalent to the statement that no points of $M$ are within distance $r$ of $p$ :

$$
\begin{aligned}
B \subset \Omega & \Longleftrightarrow|y-p|^{2} \geq r^{2} \quad \text { for all } y \in M ; \\
& \Longleftrightarrow|y-x+r \nu(x)|^{2} \geq r^{2} \quad \text { for all } y \in M ; \\
& \Longleftrightarrow|y-x|^{2}+2 r\langle y-x, \nu\rangle \geq 0 \quad \text { for all } y \in M ; \\
& \Longleftrightarrow \frac{2\langle x-y, \nu(x)\rangle}{|y-x|^{2}} \leq \frac{1}{r} \quad \text { for all } y \in M ; \\
& \Longleftrightarrow \frac{1}{r} \geq \sup \left\{\frac{2\langle x-y, \nu(x)\rangle}{|y-x|^{2}}: y \neq x\right\} .
\end{aligned}
$$

Accordingly, we define the inscribed ball curvature $\bar{k}(x, t)$ by

$$
\bar{k}(x, t)=\sup \left\{\frac{2\langle x-y, \nu(x)\rangle}{|y-x|^{2}}: y \neq x\right\},
$$

and note that this is exactly the curvature of the largest ball contained in $\Omega$ and touching at $x$. We can also define the exscribed ball curvature by taking the infimum of the same quantity (this can now be of any sign, and represents the boundary curvature of the smallest ball, halfspace or ball complement which contains $\Omega$ and touches at $x$. The key to the non-collapsing estimate for mean curvature flow is the observation that the inscribed or exscribed ball curvatures satisfy natural differential inequalities, in a viscosity sense.

If we suppose that $\varphi$ is a function which lies above $\bar{k}$ and touches at some point $\left(x_{0}, t_{0}\right)$, then as in the previous section we have two possible cases: The supremum in (113) may be attained at some $y_{0} \neq x_{0}$, or it may be attained in the limit as $y_{0}$ approaches $x_{0}$, and $\bar{k}\left(x_{0}, t_{0}\right)$ is equal to the maximum principal curvature at that point. The latter case is relatively simple, and involves the evolution equation for the second fundamental form. We suppose the former situation applies, and obtain first and second variation identities by differentiating the resulting inequality:

$$
Z(x, y, t)=2 \frac{\langle x-y, \nu(x)\rangle}{|y-x|^{2}}-\varphi(x, t) \leq 0,
$$

which holds for all $y \neq x$ and all $t \leq t_{0}$. We choose local coordinates for $M$ near $x_{0}$ and near $y_{0}$ such that the coordinate tangent vectors $\left\{\partial_{i}^{x}\right\}$ are orthonormal at $x_{0}$, and $\left\{\partial_{i}^{y}\right\}$ are orthonormal at $y_{0}$. We observe that the inscribed ball touches the hypersurface $M_{t_{0}}$ at both $x_{0}$ and $y_{0}$, implying that the tangent spaces $T_{x_{0}} M_{t_{0}}$ and $T_{y_{0}} M_{t_{0}}$ agree with the tangent spaces of the boundary sphere. In particular, the symmetry of the sphere implies that 
these tangent spaces are related by reflection, so that $R_{w}\left(T_{x_{0}} M_{t_{0}}\right)=T_{y_{0}} M_{t_{0}}$, where $R_{w}$ is the reflection given by $R_{w}(v)=v-2(v \cdot w) w$, and $w=\frac{y_{0}-x_{0}}{\left\|y_{0}-x_{0}\right\|}$. We therefore choose our local coordinates in such a way that $\partial_{i}^{y}=R_{w}\left(\partial_{i}^{x}\right)$ for each $i$ at the points $x_{0}$ and $y_{0}$. Now we begin to compute derivatives:

$$
\left(\partial_{i}^{x}+\partial_{i}^{y}\right) Z=-\frac{2}{d^{2}}\left(\left(\partial_{i}^{y}-\partial_{i}^{x}\right) \cdot(\nu(x)+(Z+\varphi) d w)+d w \cdot\left(h_{x}\right)_{i}^{p} \partial_{p}^{x}\right)-\partial_{i} \varphi .
$$

In particular, the vanishing of first derivatives at $\left(x_{0}, y_{0}, t_{0}\right)$ gives

$$
\varphi_{i}=\frac{2}{d}\left(\varphi \delta_{i}^{p}-\left(h_{x}\right)_{i}^{p}\right) \partial_{p}^{x} \cdot w
$$

at that point. Differentiating again and evaluating at $\left(x_{0}, y_{0}, t_{0}\right)$ (and using the vanishing of $Z$ and its first derivatives at that point), we obtain the following:

$$
\begin{aligned}
\sum_{i}\left(\partial_{i}^{x}+\partial_{i}^{y}\right)^{2} Z= & \frac{2}{d^{2}}\left(\left(H_{y} \nu_{y}-H_{x} \nu_{x}\right) \cdot\left(\nu_{x}+\varphi d w\right)-d w \cdot \nabla H_{x}-2 \sum_{i, q}\left(\partial_{i}^{y}-\partial_{i}^{x}\right) \cdot\left(h^{x}\right)_{i}^{q} \partial_{q}^{x}\right. \\
& \left.-2 \sum_{i} \varphi_{i} d w \cdot\left(\partial_{i}^{y}-\partial_{i}^{x}\right)-\varphi \sum_{i}\left|\partial_{i}^{y}-\partial_{i}^{x}\right|^{2}-|A|^{2} d w \cdot \nu_{x}\right) \\
& -g^{i j} \frac{\partial^{2} \varphi}{\partial x^{i} \partial x^{j}} .
\end{aligned}
$$

Differentiating in time gives the following inequality, since the rate of change of position is just $-\mathrm{H \nu}$ and the rate of change of the normal direction is $\nabla H$ under mean curvature flow:

$$
Z_{t}=\frac{2}{d^{2}}\left(\left(H_{y} \nu_{y}-H_{x} \nu_{x}\right) \cdot\left(\nu_{x}+\varphi d w\right)-d w \cdot \nabla H_{x}\right)-\varphi_{t} \geq 0 .
$$

Observe that $\nu_{x}+\varphi d w=\nu_{y}$ by the symmetry of the inscribed sphere, and that $d w \cdot \nu_{x}=-2 d^{2} \varphi$ since $Z=0$, and consequently $\nu_{y} \cdot \nu_{x}=1-\frac{d^{2} \varphi^{2}}{2}$. We also have the identity $\partial_{i}^{y}-\partial_{i}^{x}=-2\left(\partial_{i}^{x} \cdot w\right) w$. Using these we can combine (117) and (118) to produce the following inequality:

$$
\begin{aligned}
\varphi_{t}-\Delta \varphi & \leq|A|^{2} \varphi+\frac{8}{d^{2}}\left(\left(\varphi \delta_{i}^{q}-\left(h_{x}\right)_{i}^{q}\right) \partial_{i}^{x} \cdot w \partial_{q}^{x} \cdot w-4 \varphi_{i} d w \cdot \partial_{i}^{x}\right) \\
& =|A|^{2} \varphi-2\left(\left(\varphi I-h_{x}\right)^{-1}\right)^{i j} \varphi_{i} \varphi_{j},
\end{aligned}
$$

where we used the first derivative identity (116) in the last step. Since the last term is non-positive, this proves that $\bar{k}$ satisfies

$$
\partial_{t} \bar{k} \leq \Delta \bar{k}+|A|^{2} \bar{k}
$$

in the viscosity sense. Since the mean curvature $H$ is a smooth solution of the same equation, in the case where $H>0$ the ratio of $\bar{k}$ to $H$ has maximum non-increasing, and this is the non-collapsing result.

The argument above was extended to fully nonlinear evolution equations for hypersurfaces in which the speed function is either concave or convex in [16], and extended to a wider range of speed functions in [14]. The argument also works for hypersurfaces in spheres and hyperbolic spaces [18], and has 
recently been extended by Brendle to give a stronger conclusion for regions of large curvature [32], allowing also more general background spaces. The non-collapsing result for mean curvature flow is useful in understanding singularities in the mean curvature flow, particularly in the mean convex case: A powerful compactness result for non-collapsed solutions was proved by Haslhofer and Kleiner [53], and the non-collapsing estimate was a central ingredient in the recent work of Brendle and Huisken [33] on the mean curvature flow with surgery of mean-convex surfaces.

\section{The Lawson and Pinkall-Sterling conjectures and related results}

Finally, I will explain how the non-collapsing argument can be applied to minimal tori in the three-sphere, a beautiful insight of Simon Brendle [29]: Lawson conjectured in 1970 that an embedded minimal torus in the three-

dimensional sphere must be congruent to the Clifford torus $S^{1}\left(\frac{1}{\sqrt{2}}\right) \times S^{1}\left(\frac{1}{\sqrt{2}}\right)$. Of course, a minimal surface is in particular a stationary solution to the mean curvature flow, so the computations we carried out in the previous section also apply, except that the background geometry of the sphere introduces a few extra terms. The definition (113) still makes sense and has the same interpretation as before (observe that a geodesic ball in $S^{3}$ with boundary curvature $k$ is simply the intersection with $S^{3}$ of a Euclidean ball of the same boundary curvature which meets the sphere orthogonally). In the computations, the first variation equation (115) is entirely unchanged, while the second variation (117) has some extra terms coming from the fact that a second derivative of position in normal coordinates is given by $\partial_{j} \partial_{i} x=-h_{i j} \nu-g_{i j} x$, and there is a single extra term in the time inequality (118) since the evolution of the unit normal vector has an extra component in the direction normal to the sphere. This results in the following modification of (119) for mean curvature flow in a spherical background:

$$
\varphi_{t}-\Delta \varphi \leq|A|^{2} \varphi-2\left(\left(\varphi I-h_{x}\right)^{-1}\right)^{i j} \varphi_{i} \varphi_{j}+2 H-n \varphi .
$$

This was the basis for the non-collapsing result in [18]. Now specialise to the case of a minimal surface in the 3 -sphere, so that $n=2$ and $H=0$. Let $\kappa$ be the largest principal curvature, so that $|A|^{2}=2 \kappa^{2}$. Note that $\varphi I-h_{x}$ has eigenvalues $\varphi-\kappa$ and $\varphi+\kappa$, and so $\varphi I-h_{x} \leq(\varphi+\kappa) I \leq 2 \varphi I$ and we have

$$
0 \leq \Delta \varphi-\frac{|D \varphi|^{2}}{\varphi}+2\left(\kappa^{2}-1\right) \varphi
$$

proving that the inscribed ball curvature satisfies this inequality in the viscosity sense. Unlike in the case of mean curvature flow, it no longer makes sense to compare $\bar{k}$ to the mean curvature since this is zero. Brendle's insight was that one can compare instead with the largest principal curvature $\kappa$. The torus topology now comes in in a crucial way, since consideration of the holomorphic Hopf differential on a torus means that $\kappa$ is never zero in that 
case, so $\kappa$ is a smooth positive function. One can then re-write the Simons' identity in the following way:

$$
0=\Delta \kappa-\frac{|D \kappa|^{2}}{\kappa}+2\left(\kappa^{2}-1\right) \kappa
$$

The remarkable coincidence between (122) and (123) means that $\bar{k}$ and $\kappa$ can be compared: Indeed, since the surface is compact, it follows that $\bar{k}$ must be a constant multiple of $\kappa$. But it follows from the definition of $\bar{k}$ that it is greater than or equal to $\kappa$ everywhere, and indeed every embedded connected surface has a point where the inscribed ball curvature is equal to $\kappa$ (given by an extreme point on the cut locus of the distance function of the enclosed region). Therefore $\bar{k}=\kappa$ everywhere, which is an extremely rigid condition - indeed it quickly follows that the surface has a rotational symmetry with orbit circles in the direction of the principal directions corresponding to $\kappa$.

In the minimal surface case, one can repeat the same argument with the opposite choice of normal direction. The largest principal curvature in the opposite direction now has principal directions in the orthogonal directions, and we also have a rotational symmetry with circles of rotation generated by these directions. In follows that the surface is a Clifford torus.

Haizhong Li and I [17] adapted Brendle's argument to the more general constant mean curvature case, and this yielded a proof of a conjecture made in 1989 by Ulrich Pinkall and Ivan Sterling [70]: Every embedded constant mean curvature torus in the 3 -sphere is a surface of rotation. A full classification of such tori, accomplished using ODE methods, is also given in [17]. Brendle has also extended the results to allow the weaker condition of 'Alexandrov embedded' surfaces, which are immersed surfaces which lie on the boundary of an immersed 3-manifold [30]. Brendle [31] and Xuzhong Chen and myself [5] have independently extended the results to somewhat larger classes of 'Weingarten surfaces' in which a suitable function of principal curvatures is constant, deriving the same rotational symmetry result and in some cases the result that such surfaces are Clifford tori. In higher dimensions the techniques break down in general, but under the assumption of rotational symmetry (or equivalently of minimal or particular Weingarten hypersurfaces with only two values of the principal curvatures at each point) Haizhong Li, Zhijie Huang and I proved that the hypersurfaces are 'Clifford' product tori [15].

\section{References}

[1] Ben Andrews, Noncollapsing in mean-convex mean curvature flow, Geom. Topol. 16 (2012), no. 3, 1413-1418.

[2] Ben Andrews and Paul Bryan, Curvature bounds by isoperimetric comparison for normalized Ricci flow on the two-sphere, Calc. Var. Partial Differential Equations 39 (2010), no. 3-4, 419-428.

[3] Curvature bound for curve shortening flow via distance comparison and a direct proof of Grayson's theorem, J. Reine Angew. Math. 653 (2011), 179-187. 
[4] A comparison theorem for the isoperimetric profile under curve-shortening flow, Comm. Anal. Geom. 19 (2011), no. 3, 503-539.

[5] Ben Andrews and Xuzhong Chen, Embedded Weingarten tori, preprint.

[6] Ben Andrews and Julie Clutterbuck, Lipschitz bounds for solutions of quasilinear parabolic equations in one space variable, J. Differential Equations 246 (2009), no. 11, 4268-4283.

[7] , Time-interior gradient estimates for quasilinear parabolic equations, Indiana Univ. Math. J. 58 (2009), no. 1, 351-380.

[8] , Proof of the fundamental gap conjecture, J. Amer. Math. Soc. 24 (2011), no. 3, 899-916.

[9] Sharp modulus of continuity for parabolic equations on manifolds and lower bounds for the first eigenvalue, Anal. PDE 6 (2013), no. 5, 1013-1024.

[10] _ Hölder continuity of the gradient for parabolic equations in one space variable, preprint in preparation.

[11] Ben Andrews, Julie Clutterbuck, and David Shellard, Sharp height-dependent gradient estimates for heat flows, preprint in preparation.

[12] _ Modulus of continuity and gradient estimates for heat flows on evolving manifolds, preprint in preparation.

[13] _ Multi-point maximum principles and the sharp lower bound for p-eigenvalues, preprint in preparation.

[14] Ben Andrews and Mat Langford, Two-sided non-collapsing curvature flows, Annali SNS, to appear.

[15] Ben Andrews, Zhijie Huang, and Haizhong Li, Embedded Weingarten hypersurfaces with two principal curvatures in $S^{n+1}$, Recent advances in geometric analysis, Adv. Lect. Math. (ALM), Int. Press, Somerville, MA, to appear.

[16] Ben Andrews, Mat Langford, and James McCoy, Non-collapsing in fully non-linear curvature flows, Ann. Inst. H. Poincaré Anal. Non Linéaire 30 (2013), no. 1, 23-32.

[17] Ben Andrews and Haizhong Li, Embedded constant mean curvature tori in the threesphere, J. Differential Geom. 99 (2015), no. 2, 169-189.

[18] Ben Andrews, Xiaoli Han, Haizhong Li, and Yong Wei, Non-collapsing for hypersurface flows in the sphere and hyperbolic space, Annali SNS, to appear, available at http://arxiv.org/abs/1206.6240.

[19] Ben Andrews and Lei Ni, Eigenvalue comparison on Bakry-Émery manifolds, Comm. Partial Differential Equations 37 (2012), no. 11, 2081-2092.

[20] Ben Andrews and David Shellard, Modulus of continuity estimates and continuous initial data for harmonic map heat flow, preprint in preparation.

[21] Ben Andrews and Sijian Tang, Modulus of continuity for heat flows under a $C D(n, K)$ condition, preprint.

[22] Ben Andrews and Tao Su, A proof of the Smale theorem Diff( $\left.S^{2}\right) \simeq O(3)$ by curve shortening flow, preprint.

[23] Mark S. Ashbaugh and Rafael Benguria, Optimal lower bound for the gap between the first two eigenvalues of one-dimensional Schrödinger operators with symmetric single-well potentials, Proc. Amer. Math. Soc. 105 (1989), no. 2, 419-424.

[24] Mihai Bailesteanu, Xiaodong Cao, and Artem Pulemotov, Gradient estimates for the heat equation under the Ricci flow, J. Funct. Anal. 258 (2010), no. 10, 3517-3542.

[25] Dominique Bakry and Zhongmin Qian, Some new results on eigenvectors via dimension, diameter, and Ricci curvature, Adv. Math. 155 (2000), no. 1, 98-153.

[26] P. Bérard, G. Besson, and S. Gallot, Sur une inégalité isopérimétrique qui généralise celle de Paul Lévy-Gromov, Invent. Math. 80 (1985), no. 2, 295-308 (French).

[27] M. van den Berg, On condensation in the free-boson gas and the spectrum of the Laplacian, J. Statist. Phys. 31 (1983), no. 3, 623-637.

[28] Herm Jan Brascamp and Elliott H. Lieb, On extensions of the Brunn-Minkowski and Prékopa-Leindler theorems, including inequalities for log concave functions, and 
with an application to the diffusion equation, J. Functional Analysis 22 (1976), no. 4, 366-389.

[29] Simon Brendle, Embedded minimal tori in $S^{3}$ and the Lawson conjecture, Acta Math. 211 (2013), no. 2, 177-190.

[30] _ Alexandrov immersed minimal tori in $S^{3}$, Math. Res. Lett. 20 (2013), no. 3, 459-464.

[31] _ Embedded Weingarten tori in $S^{3}$, Adv. Math. 257 (2014), 462-475.

[32] _ An inscribed radius estimate for mean curvature flow in Riemannian manifolds, available at http://arxiv.org/abs/1310.3439.

[33] Simon Brendle and Gerhard Huisken, Mean curvature with with surgery of mean convex surfaces in $\mathbb{R}^{3}$, available at http://arxiv.org/abs/1309.1461.

[34] Paul Bryan, Curvature bounds via an isoperimetric comparison for Ricci flow on surfaces, available at http://arxiv.org/abs/1403.4654.

[35] Luis A. Caffarelli and Avner Friedman, Convexity of solutions of semilinear elliptic equations, Duke Math. J. 52 (1985), no. 2, 431-456.

[36] Luis A. Caffarelli and Joel Spruck, Convexity properties of solutions to some classical variational problems, Comm. Partial Differential Equations 7 (1982), no. 11, 13371379.

[37] Mu Fa Chen and Feng Yu Wang, Application of coupling method to the first eigenvalue on manifold, Sci. China Ser. A 37 (1994), no. 1, 1-14.

[38] _ Application of coupling method to the first eigenvalue on manifold, Progr. Natur. Sci. (English Ed.) 5 (1995), no. 2, 227-229.

[39] Julie Clutterbuck, Parabolic equations with continuous initial data, Australian National University, 2004, http://arxiv.org/abs/math/0504455.pdf.

[40] - Interior gradient estimates for anisotropic mean-curvature flow, Pacific J. Math. 229 (2007), no. 1, 119-136.

[41] Michael G. Crandall, Hitoshi Ishii, and Pierre-Louis Lions, User's guide to viscosity solutions of second order partial differential equations, Bull. Amer. Math. Soc. (N.S.) 27 (1992), no. 1, 1-67.

[42] James Eells Jr. and J. H. Sampson, Harmonic mappings of Riemannian manifolds, Amer. J. Math. 86 (1964), 109-160.

[43] L. Esposito, C. Nitsch, and C. Trombetti, Best constants in Poincaré inequalities for convex domains, J. Convex Anal. 20 (2013), no. 1, 253-264.

[44] L. C. Evans and J. Spruck, Motion of level sets by mean curvature. III, J. Geom. Anal. 2 (1992), no. 2, 121-150.

[45] V. A. Fateev, E. Onofri, and Al. B. Zamolodchikov, Integrable deformations of the O(3) sigma model. The sausage model., Nuclear Phys. B 406 (1993), no. 3, 521-565.

[46] David Gilbarg and Neil S. Trudinger, Elliptic partial differential equations of second order, 2nd ed., Grundlehren der Mathematischen Wissenschaften [Fundamental Principles of Mathematical Sciences], vol. 224, Springer-Verlag, Berlin, 1983.

[47] Fuzhou Gong, Huaiqian Li, and Dejun Luo, The fundamental gap conjecture: A probabilistic approach via the coupling by reflection, available at http://arxiv.org/ abs/1303.2459.

[48] Fu-Zhou Gong, Yong Liu, Yuan Liu, and De-Jun Luo, Spectral gaps of Schrödinger operators and diffusion operators on abstract Wiener spaces, J. Funct. Anal. 266 (2014), no. 9, 5639-5675.

[49] Misha Gromov, Metric structures for Riemannian and non-Riemannian spaces, Progress in Mathematics, vol. 152, Birkhäuser Boston, Inc., Boston, MA, 1999. Based on the 1981 French original [ MR0682063 (85e:53051)]; With appendices by M. Katz, P. Pansu and S. Semmes; Translated from the French by Sean Michael Bates.

[50] Richard S. Hamilton, The formation of singularities in the Ricci flow, Surveys in differential geometry, Vol. II (Cambridge, MA, 1993), Int. Press, Cambridge, MA, 1995, pp. 7-136. 
[51] _ An isoperimetric estimate for the Ricci flow on the two-sphere, Modern methods in complex analysis (Princeton, NJ, 1992), Ann. of Math. Stud., vol. 137, Princeton Univ. Press, Princeton, NJ, 1995, pp. 191-200.

[52] Isoperimetric estimates for the curve shrinking flow in the plane, Modern methods in complex analysis (Princeton, NJ, 1992), Ann. of Math. Stud., vol. 137, Princeton Univ. Press, Princeton, NJ, 1995, pp. 201-222.

[53] Robert Haslhofer and Bruce Kleiner, Mean curvature flow of mean convex hypersurfaces, available at http://arxiv.org/abs/1304.0926.

[54] Gerhard Huisken, A distance comparison principle for evolving curves, Asian J. Math. 2 (1998), no. 1, 127-133.

[55] Bernhard Kawohl, Rearrangements and convexity of level sets in PDE, Lecture Notes in Mathematics, vol. 1150, Springer-Verlag, Berlin, 1985.

[56] Alan U. Kennington, Power concavity and boundary value problems, Indiana Univ. Math. J. 34 (1985), no. 3, 687-704.

[57] _ Convexity of level curves for an initial value problem, J. Math. Anal. Appl. 133 (1988), no. 2, 324-330.

[58] J. R. King, Exact similarity solutions to some nonlinear diffusion equations, J. Phys. A 23 (1990), no. 16, 3681-3697.

[59] Nicholas J. Korevaar, Convex solutions to nonlinear elliptic and parabolic boundary value problems, Indiana Univ. Math. J. 32 (1983), no. 4, 603-614.

[60] Pawel Kröger, On the ranges of eigenfunctions on compact manifolds, Bull. London Math. Soc. 30 (1998), no. 6, 651-655.

[61] S. N. Kružkov, Nonlinear parabolic equations with two independent variables, Trudy Moskov. Mat. Obšč. 16 (1967), 329-346 (Russian).

[62] N. V. Krylov and M. V. Safonov, A property of the solutions of parabolic equations with measurable coefficients, Izv. Akad. Nauk SSSR Ser. Mat. 44 (1980), no. 1, 161175, 239 (Russian).

[63] Peter Li, A lower bound for the first eigenvalue of the Laplacian on a compact manifold, Indiana Univ. Math. J. 28 (1979), no. 6, 1013-1019.

[64] Peter Li and Shing Tung Yau, Estimates of eigenvalues of a compact Riemannian manifold, Geometry of the Laplace operator (Proc. Sympos. Pure Math., Univ. Hawaii, Honolulu, Hawaii, 1979), Proc. Sympos. Pure Math., XXXVI, Amer. Math. Soc., Providence, R.I., 1980, pp. 205-239.

[65] Shiping Liu, Gradient estimates for solutions of the heat equation under Ricci flow, Pacific J. Math. 243 (2009), no. 1, 165-180.

[66] J. Milnor, A note on curvature and fundamental group, J. Differential Geometry 2 (1968), 1-7.

[67] Aaron Naber and Daniele Valtorta, Sharp estimates on the first eigenvalue of the p-Laplacian with negative Ricci lower bound, Math. Z. 277 (2014), no. 3-4, 867-891.

[68] Lei Ni, Estimates on the modulus of expansion for vector fields solving nonlinear equations, J. Math. Pures Appl. (9) 99 (2013), no. 1, 1-16 (English, with English and French summaries).

[69] Lei Ni and Kui Wang, Isoperimetric comparisons via viscosity, preprint.

[70] U. Pinkall and I. Sterling, On the classification of constant mean curvature tori, Ann. of Math. (2) 130 (1989), no. 2, 407-451.

[71] Philipp Rosenau, On fast and super-fast diffusion, Phys. Rev. Lett. 74 (1995), 10561059.

[72] I. M. Singer, Bun Wong, Shing-Tung Yau, and Stephen S.-T. Yau, An estimate of the gap of the first two eigenvalues in the Schrödinger operator, Ann. Scuola Norm. Sup. Pisa Cl. Sci. (4) 12 (1985), no. 2, 319-333.

[73] A. S. Švarc, A volume invariant of coverings, Dokl. Akad. Nauk SSSR (N.S.) 105 (1955), 32-34 (Russian). 
[74] Daniele Valtorta, Sharp estimate on the first eigenvalue of the p-Laplacian, Nonlinear Anal. 75 (2012), no. 13, 4974-4994.

[75] Shing Tung Yau, Harmonic functions on complete Riemannian manifolds, Comm. Pure Appl. Math. 28 (1975), 201-228.

[76] Jon Wolfson, Eigenvalue gap theorems for a class of nonsymmetric elliptic operators on convex domains, available at http://arxiv.org/abs/1212.1669.

[77] Qi Huang Yu and Jia Qing Zhong, Lower bounds of the gap between the first and second eigenvalues of the Schrödinger operator, Trans. Amer. Math. Soc. 294 (1986), no. 1, 341-349.

[78] Qi S. Zhang, Some gradient estimates for the heat equation on domains and for an equation by Perelman, Int. Math. Res. Not. (2006), Art. ID 92314, 39.

[79] _ A uniform Sobolev inequality under Ricci flow, Int. Math. Res. Not. IMRN (2007), no. 17, Art. ID rnm056, 17.

[80] Jia Qing Zhong and Hong Cang Yang, On the estimate of the first eigenvalue of a compact Riemannian manifold, Sci. Sinica Ser. A 27 (1984), no. 12, 1265-1273.

Mathematical Sciences Institute, Australia National University; And Mathematical Sciences Center, Tsinghua University.

E-mail address: Ben.Andrews@anu.edu.au 